\title{
Mutual inductance instability of the tip vortices behind a wind turbine
}

\author{
Sasan Sarmast ${ }^{1,2}$, Reza Dadfar ${ }^{1}$, Robert F. Mikkelsen ${ }^{2}$, Philipp Schlatter ${ }^{1}$, \\ Stefan Ivanell ${ }^{1,3}$, Jens N. Sørensen ${ }^{2}$ and Dan S. Henningson ${ }^{1, \dagger}$ \\ ${ }^{1}$ Swedish e-Science Research Centre (SeRC), Linné FLOW Centre, KTH Mechanics, \\ Royal Institute of Technology, SE-100 44 Stockholm, Sweden \\ ${ }^{2}$ DTU Wind Energy, Denmark Technical University, 2800 Kgs. Lyngby, Denmark \\ ${ }^{3}$ Wind Energy Campus Gotland, Department of Earth Sciences, Uppsala University, \\ 62167 Visby, Sweden
}

(Received 20 November 2013; revised 22 March 2014; accepted 4 June 2014; first published online 26 August 2014)

Two modal decomposition techniques are employed to analyse the stability of wind turbine wakes. A numerical study on a single wind turbine wake is carried out focusing on the instability onset of the trailing tip vortices shed from the turbine blades. The numerical model is based on large-eddy simulations (LES) of the Navier-Stokes equations using the actuator line (ACL) method to simulate the wake behind the Tjæreborg wind turbine. The wake is perturbed by low-amplitude excitation sources located in the neighbourhood of the tip spirals. The amplification of the waves travelling along the spiral triggers instabilities, leading to breakdown of the wake. Based on the grid configurations and the type of excitations, two basic flow cases, symmetric and asymmetric, are identified. In the symmetric setup, we impose a $120^{\circ}$ symmetry condition in the dynamics of the flow and in the asymmetric setup we calculate the full $360^{\circ}$ wake. Different cases are subsequently analysed using dynamic mode decomposition (DMD) and proper orthogonal decomposition (POD). The results reveal that the main instability mechanism is dispersive and that the modal growth in the symmetric setup arises only for some specific frequencies and spatial structures, e.g. two dominant groups of modes with positive growth (spatial structures) are identified, while breaking the symmetry reveals that almost all the modes have positive growth rate. In both setups, the most unstable modes have a non-dimensional spatial growth rate close to $\pi / 2$ and they are characterized by an out-of-phase displacement of successive helix turns leading to local vortex pairing. The present results indicate that the asymmetric case is crucial to study, as the stability characteristics of the flow change significantly compared to the symmetric configurations. Based on the constant non-dimensional growth rate of disturbances, we derive a new analytical relationship between the length of the wake up to the turbulent breakdown and the operating conditions of a wind turbine.

Key words: instability, vortex interaction, wakes 


\section{Introduction}

Modern wind turbines are often clustered in wind farms and, depending on the wind direction, the turbines are fully or partially influenced by the upstream turbine wakes. Hence, an unwanted but inevitable effect is that the efficiency of the interior turbines decreases due to velocity deficits (from $5 \%$ to more than $15 \%$ efficiency loss depending on the wind farm layout, see Smith et al. 2006) and the turbulence intensity increases due to the interaction from the wakes of the surrounding wind turbines. As a consequence, dynamic loadings increase, which may excite the resonance frequency in the structural parts of the individual wind turbines and increase the fatigue loads. The turbulence created from wind turbine wakes is mainly due to the presence of the distinct tip and root vortices. In most of the situations, the organized tip/root vortex system is unstable and it eventually breaks down and forms small-scale turbulent structures. It is important to note that if a wind turbine is located in a wake consisting of stable tip and root vortices, the fatigue loading is more severe than in the case where the tip vortices have already been broken down by instability mechanisms (Sørensen 2011). Understanding the physical nature of the vortices and their dynamics in the wake of a turbine is thus important for the optimal design of a wind farm.

Joukowski (1912) was one of the first to propose a model able to describe the dominant features of a propeller. His model basically consisted of two rotating horseshoe vortices representing the tip vortices and straight root vortices. The recent study of Okulov \& Sørensen (2007) shows that the far wake of this model is unconditionally unstable.

The wake behind rotors, such as propellers, wind turbines or helicopter rotors (depending on the number of blades) can be treated as single or multiple helical vortices. The early study of a twisted helix dates back to the work by Widnall (1972) in which she provided the analytical framework to study the linear stability of a single helical vortex for inviscid flows. Widnall was among the pioneers to prove the existence of at least three different instability mechanisms. She demonstrated that helical vortices of finite core size are unstable to small sinusoidal displacements, especially as the helix pitch becomes small. The helix pitch is defined as the displacement of one complete helix turn, measured parallel to the axis of the helix. For a relatively large helix pitch, a short-wave instability mechanism may arise in the presence of perturbations with a large wavenumber, and it most likely can be observed in all the curved filaments. Furthermore, the long-wave instability may appear if the (normalized) wavenumber of the perturbations drops to less than unity. When the pitch of the helix decreases beyond a certain limit, the neighbouring filament starts to interact strongly which constitutes the underlying mechanism for the mutual inductance instability. Felli, Camussi \& Di Felice (2011) made extensive experimental investigations on propeller wakes. They noted the traces of all the three types of instability mechanisms in their experiments.

Gupta \& Loewy (1974), Bhagwat \& Leishman (2001) and Leishman, Bhagwat \& Ananthan (2004) investigated the stability of helical vortex filaments subjected to small perturbations. The vortices replicate a helicopter rotor or propeller in static thrust or axial flight condition. They identified the unstable modes in the wake structure by using a free vortex wake calculation. They found that for all perturbation wavenumbers, neutrally stable (zero growth rate) and unstable (positive growth rate) conditions exist. They reported that a maximum in the growth rates occurs at the perturbation wavenumbers equal to half-integer multiples of the number of blades, e.g. $K=N_{b}(i+1 / 2)$ for all integer $i$ where $K$ is the perturbation wavenumber and 
$N_{b}$ is the number of blades. Considering a three-blade configuration, the maximum growth thus occurs at $K=3 / 2,9 / 2,15 / 2, \ldots$. In fact, they follow a sinusoidal type of variation which has minimum values at integer multiple of the number of blades.

The aforementioned studies were performed using analytical approaches where the influence of viscosity was neglected. To account for the effects of viscosity, Walther et al. (2007) performed a series of direct numerical simulations (DNS) on the stability of helical vortices. They confirmed that the same instability mechanisms observed by Widnall can also be found in the presence of viscosity. They also identified an additional type of instability (elliptical instability) formed in the helical filaments when the radius of the curvature increases beyond a certain limit. According to this analysis, the instability has a linear phase during which small-amplitude perturbations grow, and a nonlinear phase in which the vortex core is subjected to an elliptical deformation giving rise to a local or global elliptical instability.

The stability of the tip vortices of a wind turbine has been investigated by Ivanell et al. (2010) using computational fluid dynamics (CFD) combined with the actuator line (ACL) technique. In the ACL method, which was developed by Sørensen \& Shen (2002), the presence of the blades is introduced as a body force and the flow field around the blades is determined by solving the three-dimensional Navier-Stokes equations using large-eddy simulations (LES). The resulting wake was subsequently perturbed by imposing a harmonic excitation near the tip of the blade. Analysis of the flow field indicated that the instability is dispersive and that the spatial growth arises for specific frequencies and spatial structures with wavenumbers equal to half-integer multiples of the number of blades, as was previously found in inviscid investigations similar to Gupta \& Loewy (1974). Bhagwat \& Leishman (2001) and Leishman et al. (2004), and discussed above.

The pairing instability in the wake of a wind turbine was first seen in the smoke visualization of Alfredsson \& Dahlberg (1979). Recent experiments by Felli et al. (2011) and Leweke et al. (2013) as well as numerical studies by Widnall (1972) and Ivanell et al. (2010) have confirmed that the mutual inductance instability leads to vortex pairing in the rotor wake, and they indicate that the vortex pairing is the primary cause of wake destabilization. The vortex pairing is a result of the vortex-induced velocities in a form that it is analogous to the leapfrogging motion of two inviscid vortex rings (Jain et al. 1998). This phenomenon occurs in a row of equidistant identical vortices, whereby amplifications of small perturbations cause the vortices to oscillate in such a way that neighbouring vortices approach each other and start to group in pairs. Lamb (1932) has analysed the stability of single and double rows of identical vortices in two dimensions, representing the parallel helical vortices in three dimensions. He found that the maximum non-dimensional temporal growth rate can reach up to $\sigma=\pi / 2$ in both setups. Here we identify the non-dimensional instability growth rate of the pairing as $\sigma=\tilde{\sigma}\left(2 h^{2} / \Gamma\right)$, where $\tilde{\sigma}$ is the dimensional spatial growth rate, $h$ and $\Gamma$ are the helix pitch and circulation of the vortices, respectively. Levy \& Forsdyke (1928) have treated analytically the stability of an array of axisymmetric vortex rings. They found that the two parameters of pitch and core size influence the growth rate. However, for small helix pitch and core sizes the maximum growth rate approaches the value of $\sigma=\pi / 2$. The analytical stability analysis of Widnall (1972) using a single twisted helix reveals that the most unstable perturbations have an out of phase oscillation between the neighbouring vortex spirals with the non-dimensional growth close to $\sigma=\pi / 2$. Ivanell et al. (2010) and Leweke et al. (2013) also reached a similar conclusion that the highest growth rate perturbations have a non-dimensional growth rate close to $\sigma=\pi / 2$, although in their studies it was the spatial growth rate that was found. 
The present study focuses on the stability properties of tip vortices and the mechanisms leading to vortex pairing. The basic wake behind a horizontal axis wind turbine is computed by combining an unsteady incompressible Navier-Stokes solver (EllipSys3D) and the ACL method. This work is a continuation of the work previously performed by Ivanell et al. (2010). We generalized the excitation sources by perturbing the flow using low-amplitude harmonic or stochastic excitations near the tip of the blades. Different setups including one-third and full polar domain configurations are studied, and the unstable modes are extracted using two modal decomposition techniques, i.e. proper orthogonal decomposition (POD) (Lumley 1970; Sirovich 1987) and dynamic mode decomposition (DMD) (Rowley et al. 2009; Schmid 2010). In the present study, the mutual inductance is most prominent, but also traces of the long-wave instability mechanism could be observed when the structures representing the short-wave instability are not part of the solutions.

This paper is organized as follows: in $\S 2$, the simulation setup and ACL method are described. A brief overview of modal decomposition techniques is presented in $\S 3$. The modal analysis of the flow behind a wind turbine is carried out in $\S 4$. The main results are highlighted in $\S 5$. A novel method for determining the near-wake length is proposed in $\S 6$ and the paper ends with a summary of the main conclusions in $\S 7$. In addition, the modal decomposition techniques and their convergence characteristics are discussed in detail in the appendix A.

\section{Problem setup}

The so-called ACL method, introduced by Sørensen \& Shen (2002), is a fully three-dimensional and unsteady aerodynamic model for simulating wind turbine wakes. In this method, the flow around the rotor is governed by the three-dimensional incompressible Navier-Stokes equations, while the influence of the blades on the flow field is approximated by a body force. The force is determined using the blade element method (BEM) combined with tabulated airfoil data. For each blade, the body force is distributed radially along a line representing the blade of the wind turbine. At each point of the line, the force is smeared among neighbouring nodes with a three-dimensional Gaussian distribution in order to avoid the numerical singular behaviour and mimic the chord-wise pressure distribution (for more details, see Mikkelsen 2003). The ACL method is implemented into the EllipSys3D code developed by Michelsen (1994) and Sørensen (1995). The EllipSys3D code is based on a multiblock/cell-centred fourth-order finite volume discretization of the incompressible Navier-Stokes equations. The code is formulated in primitive variables, i.e. in pressure and velocity variables, in a collocated storage arrangement. Rhie/Chow interpolation is used to avoid odd/even pressure decoupling. Using the Cartesian coordinate system, the governing equations are formulated as

$$
\begin{aligned}
& \frac{\partial \tilde{u}_{i}}{\partial \tilde{t}}+\frac{\partial \tilde{u}_{i} \tilde{u}_{j}}{\partial \tilde{x}_{j}}=-\frac{1}{\rho} \frac{\partial \tilde{p}}{\partial \tilde{x}_{i}}+\tilde{f}_{b o d y, i}+2 \mathscr{E}_{i j k} \Omega_{j} \tilde{u}_{k}+\frac{\partial}{\partial \tilde{x}_{j}}\left[\left(v+v_{t}\right)\left(\frac{\partial \tilde{u}_{i}}{\partial \tilde{x}_{j}}+\frac{\partial \tilde{u}_{j}}{\partial \tilde{x}_{i}}\right)\right], \\
& \frac{\partial \tilde{u}_{i}}{\partial \tilde{x}_{i}}=0,
\end{aligned}
$$

where $i, j \in\{1,2,3\}$ represent the three orthogonal coordinate directions, $\tilde{u}_{i}$ is the dimensional velocity vector, $\tilde{p}$ is the pressure, $\tilde{f}_{b o d y, i}$ represents the dimensional ACL forces, $2 \mathscr{E}_{i j k} \Omega_{j} \tilde{u}_{k}$ is the Coriolis force where $\mathscr{E}_{i j k}$ is the permutation matrix, $\Omega_{j}$ is the rotational frequency, $v$ is the kinematic viscosity and $v_{t}$ is the eddy viscosity. 


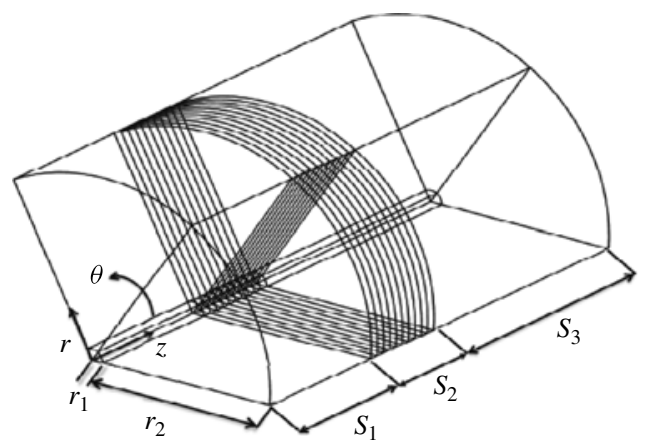

\begin{tabular}{cc}
\hline Parameter & Length (in $R$ ) \\
$r_{1}$ & 1.7 \\
$r_{2}$ & 13.3 \\
$S_{1}$ & 18.4 \\
$S_{2}$ & 8 \\
$S_{3}$ & 17.6 \\
\hline
\end{tabular}

FIGURE 1. Schematic of the multi-block mesh topology; $r_{1}$ and $S_{2}$ represent the near domain where the grid is distributed equidistantly in all directions. In regions $r_{2}, S_{1}$ and $S_{3}$, the grids are stretched towards the outer boundaries in both axial and radial directions. The ACL is discretized by 97 grid points to resolve the blade accurately. The flow direction corresponds to the positive $z$-axis and the rotor is located inside the near domain at $z=19$. Only one third (i.e. $120^{\circ}$ ) of the polar domain is shown here.

We employ LES in which the large scales are resolved and the small scales are modelled by an eddy-viscosity-based subgrid-scale model developed by Ta Phuoc (1994). The computations are performed in the Cartesian system while some of the results are presented in polar coordinates. The computational domain is discretized in an axisymmetric $360^{\circ}$ polar grid with 85 million grid points. In the vicinity of the ACLs, referred to as the near domain, approximately 50 million grid points are used in order to capture the gradients and to resolve the near-wake dynamics. The simulations are performed in a rotating frame of reference where the ACLs are stationary. Regarding the boundary conditions, the velocity at the inlet is assumed to be uniform in the axial direction. A convective outflow boundary condition is used at the outlet. Figure 1 shows the computational domain where the outer cylindrical domain boundary is chosen far from the rotor to ensure very low blockage effects. Note that all the simulation parameters are normalized by the inlet velocity $U_{0}$ and the blade length $R$ and all non-dimensional quantities are denoted without a tilde.

The quality of the numerical results using the ACL method depends on the accuracy of the airfoil data. In this sense, the Tjæreborg turbine consisting of NACA44xx airfoils is an appropriate test case since it has been extensively tested and verified, see Mikkelsen (2003) and Troldborg (2008), and the blade data are publicly available (Øye 1991). The current simulations are performed based on the Tjæreborg wind turbine operating at the optimum power condition (pressure coefficient $C_{p}=0.49$ ) with a wind speed of $U_{0}=10 \mathrm{~m} \mathrm{~s}^{-1}$ and tip speed ratio of $\lambda=R \Omega / U_{0}=7.07$ where $\Omega$ is the angular velocity. In addition, the simulations are conducted at Reynolds number $\left(R e=U_{0} R / v\right)$ of $10^{5}$ where $v$ is the kinematic viscosity of air. Sørensen, Shen \& Munduate (1998), Mikkelsen (2003) and Troldborg (2008) mentioned that due to the absence of boundary layers, above a certain minimum the Reynolds number has only a minor effect on the overall wake behaviour.

To proceed with the simulation, first, a steady flow is obtained while integrating without disturbances up to simulation time $t=\tilde{t}\left(U_{0} / R\right)=250$ (figure $2 a$ ). This time is analogous to the time required for the flow to pass roughly six times through the domain. This flow state can be considered as the base flow (equilibrium solution of the governing equations). In the present case, it can be obtained by time integration 

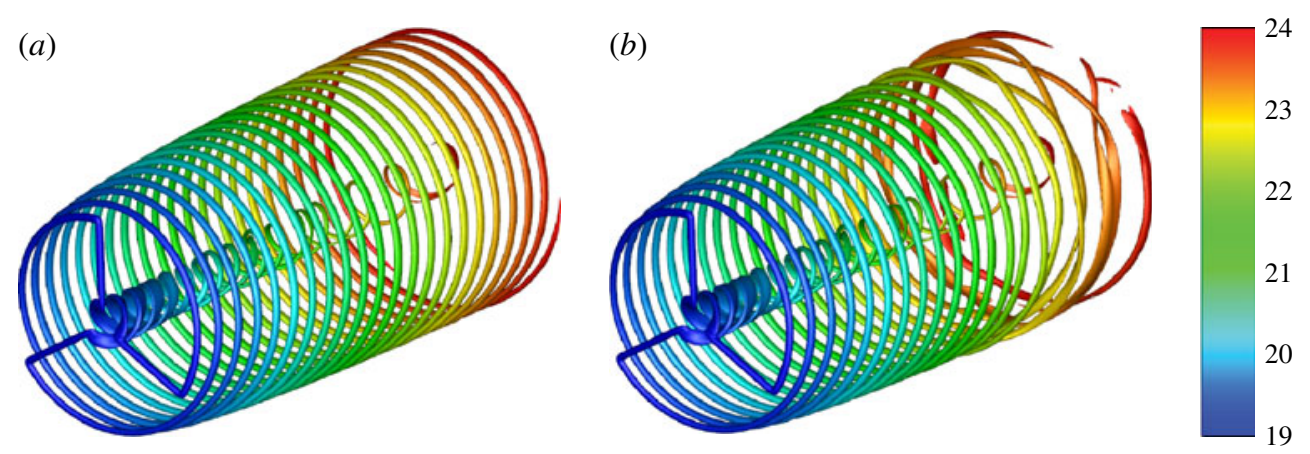

FIGURE 2. Isosurfaces of vorticity magnitude coloured by axial position $z$ where the vorticity magnitude (enstrophy) is given by $\|\omega\|=\left(\omega_{r}^{2}+\omega_{t}^{2}+\omega_{z}^{2}\right)^{1 / 2}$. (a) Basic steady state (base flow) and $(b)$ helical vortices breaking down under the influence of the stochastic noise excitation.

of the unforced equations because in the absence of forcing (noise excitation) all the unsteady perturbations will be washed out of the system, confirming the convectively unstable nature of the flow at hand. Then, additional perturbations are introduced slightly downstream of the tip of the blade. These perturbations trigger the instability mechanisms which result in the wake breakdown (figure $2 b$ ). When the flow reaches its fully developed non-stationary condition at $t=300$, we start to collect snapshots used in the data analysis with the time separation $\Delta t=25 \times 10^{-3}$.

A controlled way to introduce the additional perturbations on the helical vortices is to apply a low-amplitude external excitation (body force) on top of the base flow. The spatial shape of the body force is assumed to be a Gaussian distribution while the corresponding temporal part is considered as a low-pass-filtered stochastic noise or harmonic excitation,

$$
f_{b o d y}(x, y, z)=a \exp \left(-\gamma_{x}^{2}-\gamma_{y}^{2}-\gamma_{z}^{2}\right) g(t),
$$

where

$$
\gamma_{x}=\frac{x-x_{0}}{\eta_{x}}, \quad \gamma_{y}=\frac{y-y_{0}}{\eta_{y}}, \quad \gamma_{z}=\frac{z-z_{0}}{\eta_{z}},
$$

and $\left(x_{0}, y_{0}, z_{0}\right)$ is the centre of the Gaussian distribution. The parameters $\left(\eta_{x}, \eta_{y}, \eta_{z}\right)$ represent the size of the spatial shape of the forcing; $a$ denotes the amplitude and $g(t)$ indicates the temporal part of the forcing. In this study, the force is applied only in the axial direction and the amplitude is tuned $(a=0.005)$ to obtain the perturbation fields. The generated noise is considered as a model of free-stream turbulence with turbulence intensity $I=0.1 \%$ near the rotor tip. The temporal part of the forcing is assumed to be either the harmonic excitation for the specific frequencies or stochastic forcing with uniform energy distribution over all frequencies lower than a certain cutoff frequency (figure 3). The cutoff frequency for the stochastic forcing is chosen such that all the physically relevant frequencies lie in the flat region of the spectrum so that all the frequencies are given the same chance to grow and the most unstable waves dominate the wake. For further details on the computation of the low-passfiltered noise, we refer to Chevalier et al. (2007) and Schlatter \& Örlü (2012).

We consider a subdomain of the complete computational domain to perform the modal analysis, in order to reduce the size of the computations and focus on the 


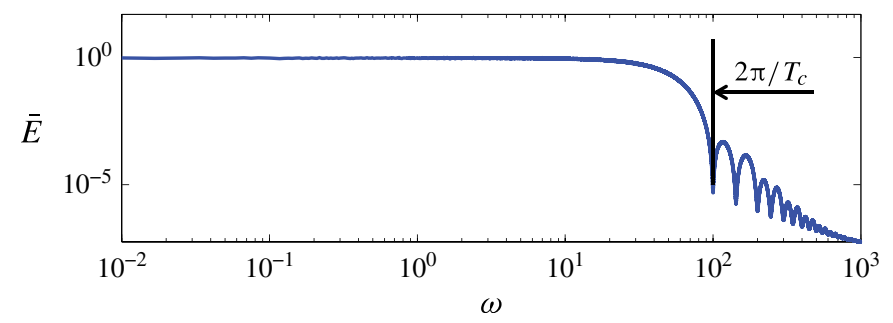

FIGURE 3. Normalized power spectrum of the low-pass-filtered noise.

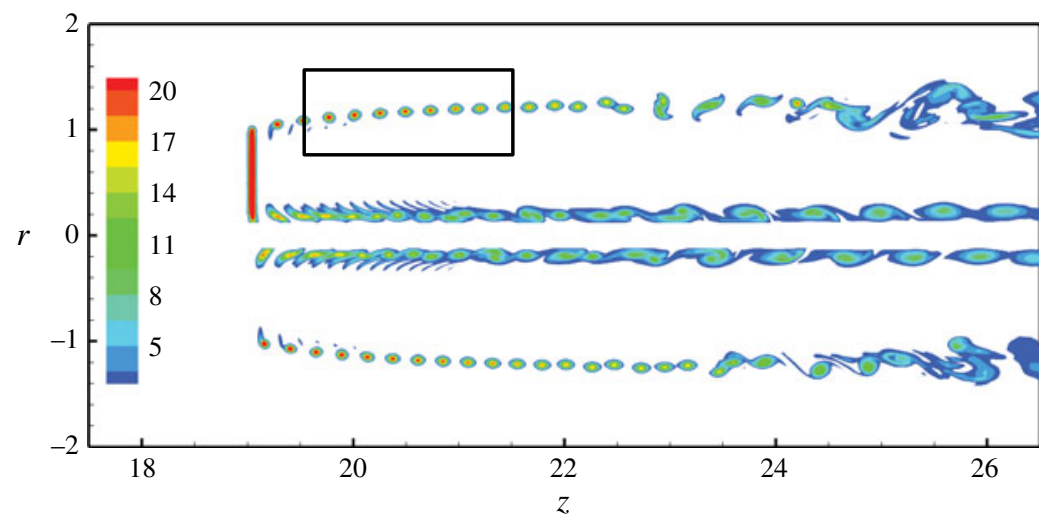

FIGURE 4. Contours of vorticity magnitude in an $r, z$-plane cut show the development of the tip spiral instability. The black box marks the radial and axial lengths of the linear subdomain considered.

interesting part relevant to the physics in the flow. It is important to position the subdomain in the wake such that we can study the onset of instability. The nonlinear effects in the wake breakdown and the turbulent wake are outside the subdomain. Thus, we consider a subdomain where the perturbation development is linear and the magnitude of the velocity of the perturbations remains small compared to the base flow. In this region, based on the observations by Tangler, Wohlfeld \& Miley (1973), the instability appears to begin at the location where the rotor wake has undergone a maximum radial expansion. In figure 4 , a cutoff of the chosen toroidal subdomain is depicted. In general depending on the tip speed ratio, the tip and root vortices may interact. In the present study due to the proximity of the root vortices, they break down faster than the tip vortices and there is no interaction between root and tip vortices. Therefore, root vortices are not included in our analysis.

Based on the grid configuration and the applied external excitation, we study several configurations (see table 1). In the first group (cases A and B), we assumed a symmetry condition in the geometry and calculate only one third of the polar domain (i.e. $120^{\circ}$ ), using periodic boundary conditions on the lateral sides. Two additional cases (cases $\mathrm{C}$ and $\mathrm{D}$ ) with the full-domain (i.e. $360^{\circ}$ ) configuration are also studied; first three identical excitation sources are located near the tip of each blade, leading to the same symmetry between the three blades as in the $120^{\circ}$ case. In the last simulation, case $\mathrm{E}$, the full polar domain is used and three uncorrelated (i.e. asymmetric) excitation sources are imposed near the tip of each blade. 
Case Polar domain (deg.)

Perturbation type
Harmonic
Noise
Harmonic
Noise
Noise

Perturbation correlation

$\begin{array}{ll}\text { A } & 120 \\ \text { B } & 120 \\ \text { C } & 360 \\ \text { D } & 360 \\ \text { E } & 360\end{array}$

Correlated due to symmetry

Correlated due to symmetry

Correlated

Correlated

Uncorrelated

TABLE 1. The configurations studied.

\section{Theoretical background}

The coherent features of the wind turbine wakes are identified by modal decomposition techniques in order to describe the underlying mechanism of wake breakdown. One benefit of modal decomposition is the possibility of reducing the large-scale dynamics to fewer degrees of freedom. This section is focused on providing a brief theoretical background of two modal decomposition approaches: POD (Lumley 1970; Sirovich 1987) and DMD (Rowley et al. 2009; Schmid 2010).

\subsection{Modal decomposition}

To extract coherent motion from a given dataset, we consider a flow with equidistantly sampled (with spacing $\Delta t$ ) velocity fields; the sequence of $m$ discretized flow fields $\boldsymbol{u}_{j}=\boldsymbol{u}\left(\boldsymbol{x}_{i}, t_{j}\right) \in \mathbb{R}^{n}, t_{j}=j \Delta t, j=0,1, \ldots, m-1$ are assembled column-wise as

$$
\boldsymbol{U}_{m}=\left[\boldsymbol{u}_{0}, \boldsymbol{u}_{1}, \ldots, \boldsymbol{u}_{m-1}\right] \in \mathbb{R}^{n \times m},
$$

where $n$ is the total number of degrees of freedom at one time instant and is equivalent to the number of grid points multiplied by the number of velocity components. This number is usually large compared to the number of snapshots $m$ in the flow problem, $n \gg m$. In modal decomposition, we attempt to split the flow dynamics into spaceand time-dependent parts; this is achieved by expanding the velocity field into spatial basis functions (the modes) $\phi_{k}=\phi_{k}\left(\boldsymbol{x}_{i}\right), k=0, \ldots, m-1$, and the corresponding temporal coefficients (amplitudes) $\boldsymbol{a}_{k}=\boldsymbol{a}_{k}\left(t_{j}\right)$, as

$$
\boldsymbol{u}\left(\boldsymbol{x}_{i}, t_{j}\right)=\sum_{k=0}^{m-1} \phi_{k}\left(\boldsymbol{x}_{i}\right) \boldsymbol{a}_{k}\left(t_{j}\right) \quad \forall j \text { or } \boldsymbol{U}_{m}=\boldsymbol{X} \boldsymbol{T},
$$

where the matrix $\boldsymbol{T}$ contains the temporal coefficients $\boldsymbol{a}_{k}$ and $\boldsymbol{X}$ is the collection of the spatial basis $\phi_{k}$. Since only modes describing the coherent motions are significant, we can usually reconstruct the flow with good accuracy using fewer modes. Mathematically, a variety of modal decompositions have been derived, two of which are used in this paper, namely POD and DMD. POD (see § A.1) can be considered as a purely statistical method where the modes are obtained from minimization of the residual energy between the snapshots and its reduced linear representation; the spatial and temporal modes are constructed to be mutually orthogonal, see Sirovich (1987). In DMD (see $\$ 3.2$ below and $\S$ A.2), on the other hand, it is assumed that the snapshots are generated by a dynamical system; the extracted modes are characterized by a specific frequency and growth rate. In fact, in POD, the modes are related to the eigenfunctions of the two-point spatial correlation matrix, whereas in DMD, the dynamic modes are computed from the eigenfunctions of the approximated linear Koopman operator. 


\subsection{Dynamic mode decomposition}

From a mathematical point of view, dynamic modal decomposition (DMD) is an Arnoldi-like method which has many similarities to the algorithm presented by Prony (1795) two centuries ago. Without explicit knowledge of the dynamical operator, it extracts frequencies, growth rates, and their related spatial structures (modes). DMD splits the flow into different modes that independently oscillate at a certain frequency. Rowley et al. (2009) presented the theoretical basis of the DMD to compute the Koopman expansion from a finite sequence of flow fields (snapshots); Schmid (2010), among other considerations, provided an improvement towards a more stable implementation of the DMD algorithm. The current DMD method has a number of advantages: it is possible to analyse the flow even when nonlinear interactions are present in parts of the domain. At the same time, the obtained modes are similar to linear global modes in those regions where the amplitudes are small. Moreover, the implementation of the method is comparably straightforward as only snapshots are needed, and computationally efficient, if both POD and DMD modes are required. To compute DMD, we consider a sufficiently long, but finite time series of snapshots (see (3.1)). We assume a linear mapping that associates the flow field $\boldsymbol{u}_{j}$ to the subsequent flow field $\boldsymbol{u}_{j+1}$ such that

$$
\boldsymbol{u}\left(\boldsymbol{x}_{i}, t_{j+1}\right)=\boldsymbol{u}_{j+1}=\mathrm{e}^{\tilde{\boldsymbol{A}} \Delta t} \boldsymbol{u}_{j}=\boldsymbol{A} \boldsymbol{u}_{j} .
$$

Hence, it is possible to write

$$
\boldsymbol{u}\left(\boldsymbol{x}_{i}, t_{j}\right)=\sum_{k=0}^{m-1} \phi_{k}\left(\boldsymbol{x}_{i}\right) \boldsymbol{a}_{k}\left(t_{j}\right)=\sum_{k=0}^{m-1} \phi_{k}\left(\boldsymbol{x}_{i}\right) \mathrm{e}^{\mathrm{i} \omega_{k} j \Delta t}=\sum_{k=0}^{m-1} \phi_{k}\left(\boldsymbol{x}_{i}\right) \lambda_{k}^{j},
$$

where $\mathrm{i} \omega_{k}$ and $\lambda_{k}$ are the eigenvalues of the matrices $\tilde{\boldsymbol{A}}$ and $\boldsymbol{A}$, respectively, and $\phi_{k}$ are the corresponding eigenvectors. We also have the relation linking the eigenvalues $\lambda_{k}$ and the more familiar complex frequencies $\mathrm{i} \omega_{k}$

$$
\lambda_{k}=\mathrm{e}^{\mathrm{i} \omega_{k} \Delta t} .
$$

It is further possible to write $\phi_{k}=\boldsymbol{v}_{k} d_{k}$ where $\boldsymbol{v}_{k}^{\mathrm{T}} \boldsymbol{M} \boldsymbol{v}_{k}=1$. We define $d_{k}$ as the amplitude and $d_{k}^{2}$ as the energy of the dynamic mode $\phi_{k}$ (see $\S$ A.2).

\section{Results from modal decomposition}

\subsection{Description of employed data set}

To ensure the convergence of the method ( $\mathrm{A} .4$ ), we assume different numbers of snapshots and sampling intervals to decrease the residual below a certain limit. The choice of the sampling interval is dependent on the highest relevant frequency in the flow. According to the Nyquist criterion, with a sampling interval $\Delta t$ we can resolve the highest frequency $f=(2 \Delta t)^{-1}$. Increasing the number of snapshots with a constant sampling interval extends the total time of the snapshots which is associated with the lowest resolved frequency in the flow. We remark that the DMD method is subject to the Nyquist frequency criterion for high frequencies, but it can determine modes with frequencies even lower than the one given by the total time spanned by the snapshots, see Chen, Tu \& Rowley (2012). However, in this study we ensure that the longest relevant period in the flow is bounded by the accumulated snapshot period. 


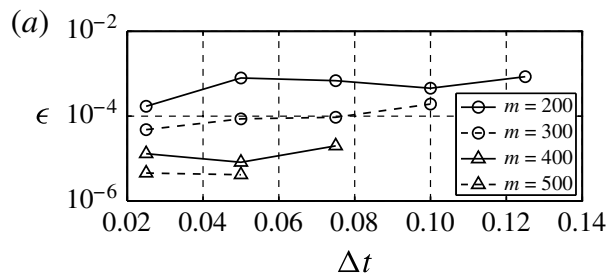

(b)

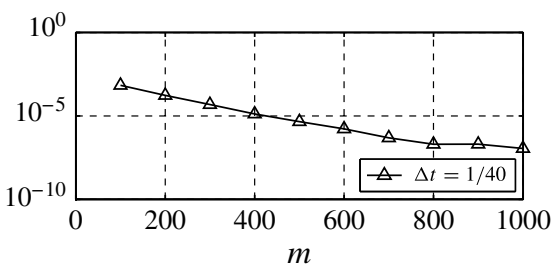

FIGURE 5. Residual norm (least-square-problem residual) of the DMD as a function of (a) sampling interval with different numbers of snapshots and $(b)$ the number of snapshots with constant sampling interval for $\Delta t=1 / 40$.

The variation of the DMD residual for different datasets is presented as a function of sampling interval and number of snapshots in figure 5(a). The residual is quite sensitive to the number of snapshots. In fact, as we increase the number of snapshots from $m=300$ to $m=500$, the residual decreases. However, the variation of the residual is quite insensitive to the sampling interval. In particular, if we decrease the sampling interval from $\Delta t=1 / 20$ to $\Delta t=1 / 40$ for $m=500$ snapshots, the residual does not change. Based on that, we choose $\Delta t=1 / 40$ as the sampling interval of the snapshots for the current investigation. To select an appropriate number of snapshots, we keep a constant sampling interval $\Delta t=1 / 40$ and report the variation of the residual as we change the number of snapshots (figure $5 b$ ). The residual in this case decreases as the number of snapshots increases and reaches a plateau for $m=800$ after which it does not change significantly. Hence we consider $m=896$ snapshots for this study.

The convergence of the data set analysis has also been well investigated for the POD procedure. The convergence is achieved due to further reduction in the time interval between two consecutive snapshots and increasing the number of snapshots. The results show that taking $m=896$ snapshots with sampling interval $\Delta t=1 / 40$ ensures the convergence of the modes and their time coefficients.

\subsection{Spectra and spatial modes}

In this paper, the Welch method together with a Hamming window is implemented in order to deal with the noisy data introduced by the stochastic excitation (see $\S$ A.3). In total $m=1344$ snapshots are used. We split the snapshots into two segments, each segment includes $m=896$ snapshots with $50 \%$ overlap. For each segment the DMD analysis with a Hamming window is performed and the resulting power spectral density is averaged. Before performing DMD on the fluctuating part, we subtract the mean flow from the dataset. Since the current computational domain lies in the linear region of the wind turbine wakes and the flow dynamics is statistically steady, we do not expect to find modes with temporal growth rate in the results. In fact, the eigenvalues resulting from the modified companion matrix, $\lambda_{k}$, are complex conjugates and they all lie on the unit circle (not shown here), $\left|\lambda_{k}\right|=1$. This behaviour is expected once we subtract the mean flow (Chen et al. 2012). The complex eigenvalues, $\lambda_{k}$, are then logarithmically mapped onto the complex plane, i.e. $\mathrm{i} \omega_{k}=\log \left(\lambda_{k}\right) / \Delta t$ with $\Delta t=1 / 40$ such that the imaginary part represents an exponential growth rate and the real part represents the temporal frequency.

The results for the power spectral density for the three different configurations (onethird symmetric, full-domain symmetric and full-domain asymmetric), are presented in figure 6 . For the $120^{\circ}$ and $360^{\circ}$ symmetric configurations, as expected, the dynamics 

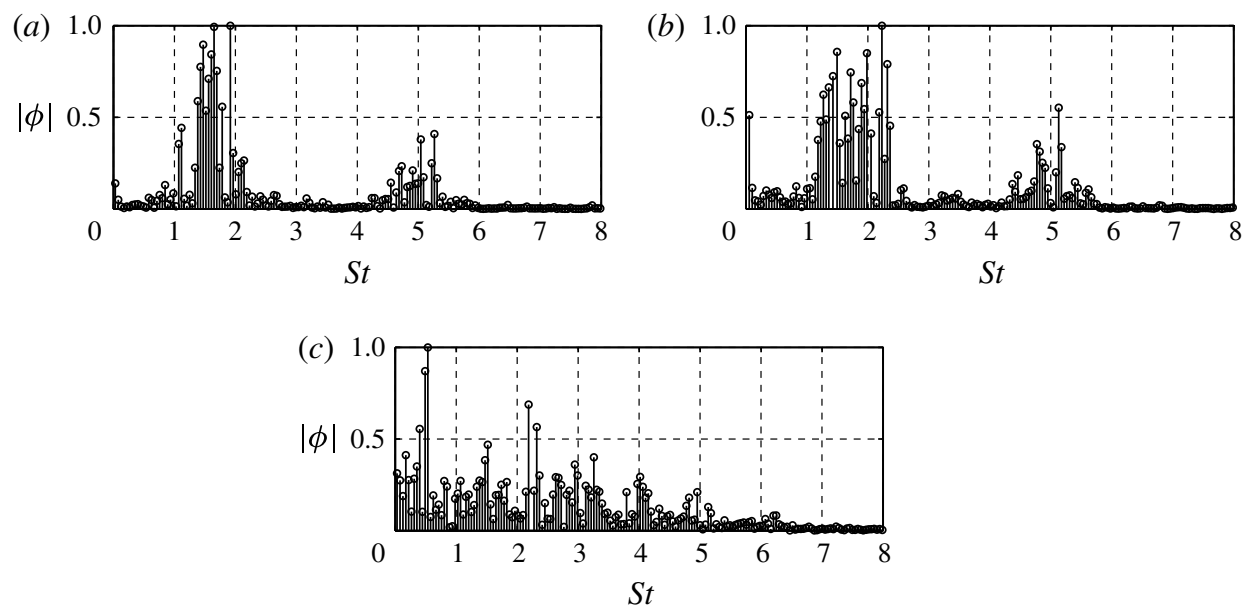

FIGURE 6. Amplitude distribution of the averaged dynamic modes as a function of Strouhal number of $(a)$ symmetric, one-third domain (case B table 1), (b) symmetric, full domain (case D) and (c) asymmetric, full domain (case E).

of the flow is quite similar. In both cases, two dominant frequencies can be identified in the flow; one frequency around $S t \approx 2$ and the other around $S t \approx 5$. The nondimensional frequency is defined as a Strouhal number $S t=f R / U_{\infty}$. On the other hand, the power spectrum is quite different for the asymmetric setup (figure $6 c$ ). In fact, not only are the two previous dominant frequencies $(S t \approx 2$ and $S t \approx 5)$ observed in the spectra, additional dominant frequencies (around $S t=\{0.5,3,4,6\}$ ) are also noticeable.

The same dominant frequencies can also be identified in the POD analysis. The POD decomposes the dynamics of the flow into a set of orthogonal modes with corresponding time evolutions. The dominant frequencies in each mode can be identified by computing the power spectral density of the temporal coefficient. Figure 7 depicts the power spectral density for both configurations: symmetric and asymmetric. Similar to DMD, for the symmetric case, two dominant frequencies $S t \approx 2$ and $S t \approx 5$ can be identified in the flow; the frequency $S t \approx 2$ is dominant among the most energetic modes $2-3$ and 6-7 while the frequency $S t \approx 5$ has considerable energy in the modes 4-5 and 8-9. Mode 1 is not shown in the figure since it represent the mean flow and does not make any contribution to the dynamics of the fluctuations. Figure 7(b) shows the power spectral density for the asymmetric configuration. In this case a broader range of dominant frequencies can be distinguished. In fact, the leading most-energetic modes have dominant frequencies $S t \approx\{0.5,2,3,4,5,6\}$.

Figure 8 shows the distribution of energy of the fluctuations among the POD modes. For each mode the contribution of energy to the total energy of the fluctuations is presented for both configurations: symmetric and asymmetric. This share is computed as the percentage of the energy with respect to the total energy of the fluctuating parts. The total energy of the fluctuating part is obtained by summing up the energy of all the modes (summation of the eigenvalues) excluding the first one. We exclude the first mode (not shown here) since it represents the energy of the mean flow with an approximately constant time coefficient. A closer look at the energy of the modes reveals that the leading energetic ones appear in pairs. In fact, the eigenvalues of these modes are the same (the energy of the two modes is equal), their spatial structure is similar but with a phase shift and their time coefficients have the same 

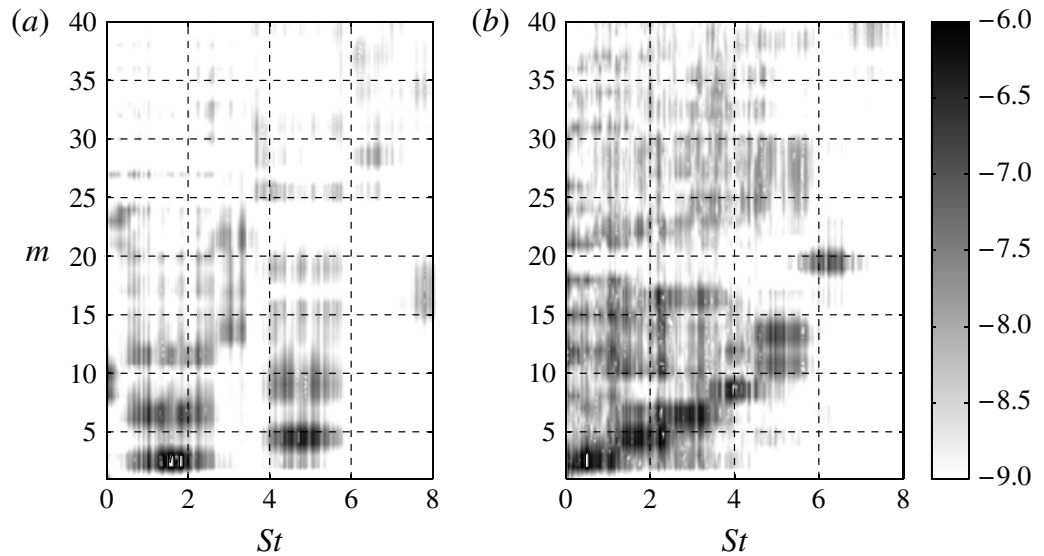

FIgure 7. Power spectral density (in logarithmic scale) of the first 40 POD time coefficients of (a) symmetric one-third domain (case B in table 1) and $(b)$ asymmetric configuration (case E). The POD modes larger than mode 20 do not show a significant energy contribution to the dynamics of the fluctuations.
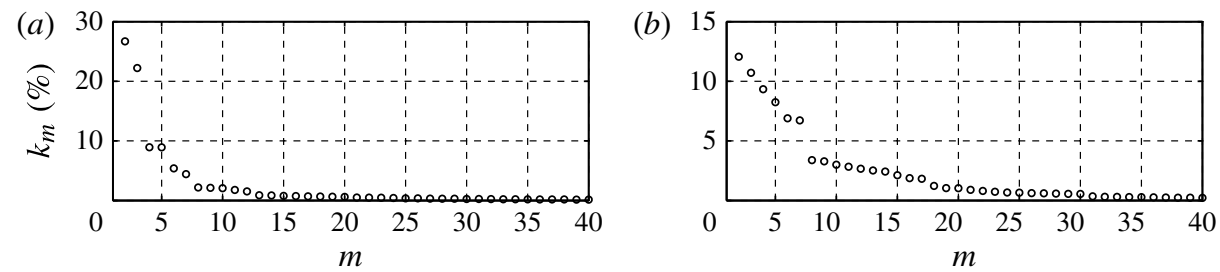

FIgURE 8. The energy contribution of the modes $2-40$ to the total energy of the fluctuations of (a) symmetric one-third domain (case B in table 1) and (b) asymmetric full-domain configuration (case E).

frequency content. Any pair of these POD modes represents the dynamics of a wave-like travelling structure (see e.g. Rempfer \& Fasel 1994).

The spatial shapes of these travelling structures give valuable information about the coherent structures in the flow. In DMD, the spatial structures associated with dominant frequencies and for both configurations, symmetric and asymmetric, are depicted in figures 9 and 10. In the symmetric case, the dominant modes contain three or nine lobes in one revolution corresponding to wavenumbers $K \approx 3 / 2$ and $K \approx 9 / 2$. Here, $K$ is defined as the number of complete waves over a $360^{\circ}$ turn along the spiral. These results are in agreement with the observations by Ivanell et al. (2010) for a three-bladed rotor. However, if we apply the asymmetric stochastic forcing at the tips of the blades, a different behaviour is observed. In fact, in this case, six dominant group of modes can be identified which correspond to wavenumbers $K \approx\{1 / 2,3 / 2,5 / 2,7 / 2,9 / 2,11 / 2\}$. Among these, only the wavenumbers $K \approx\{3 / 2,9 / 2\}$ were previously observed in the symmetric case. The same conclusion can be drawn from the POD analysis. The spatial structures associated with the dominant frequencies for both configurations are shown in figures 11 and 12. Similar to the DMD, for the symmetric case, the spatial structures of the energetic modes, associated with $S t \approx 2$ and $S t \approx 5$, have three and nine lobes corresponding to the 

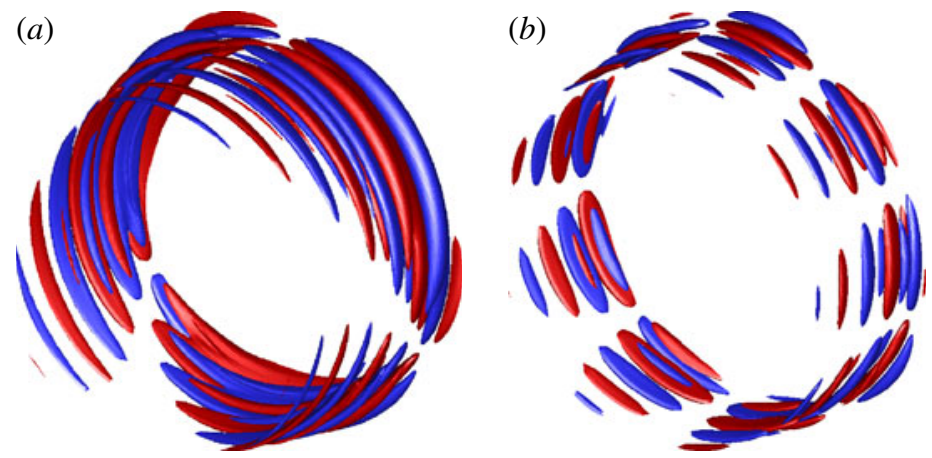

FIGURE 9. Selected dynamic modes of the symmetric, one-third domain (case B in table 1): (a) first group $K \approx 3 / 2, S t=1.95$ and $(b)$ second group $K \approx 9 / 2, S t=5.15$.
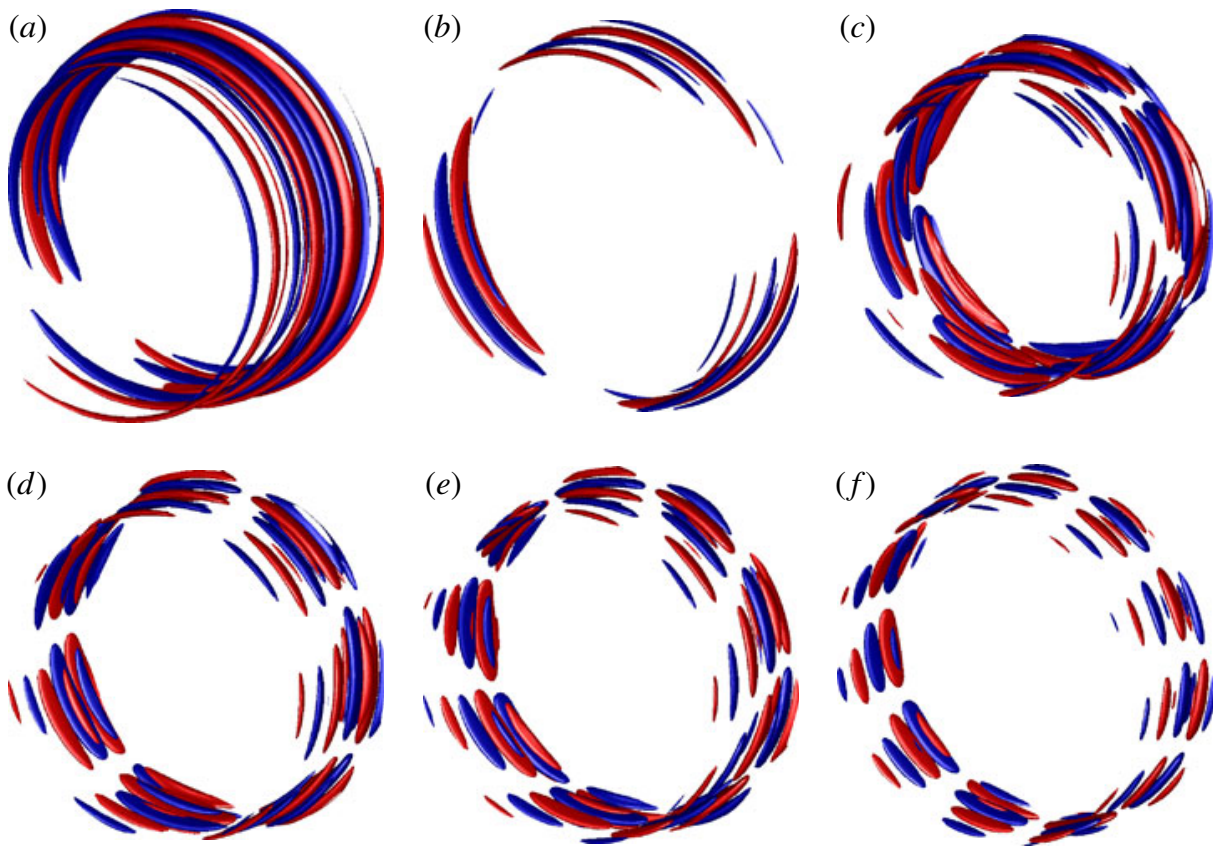

FIGURE 10. Selected dynamic modes of the asymmetric, full-domain (case E in table 1): (a) first group $K \approx 1 / 2, S t=0.51,(b)$ second group $K \approx 3 / 2, S t=2.22,(c)$ third group $K \approx 5 / 2, S t=3.16,(d)$ fourth group $K \approx 7 / 2, S t=3.90$, (e) fifth group $K \approx 9 / 2, S t=4.84$ and $(f)$ sixth group $K \approx 11 / 2, S t=6.21$.

wavenumbers $K \approx 3 / 2$ and $K \approx 9 / 2$, respectively. However, for the asymmetric case, the spectrum is quite different. In fact, in this case, the energetic modes contain spatial wavenumbers $K \approx\{1 / 2,3 / 2,5 / 2,7 / 2,9 / 2,11 / 2\}$ where the presence of additional spatial wavenumbers $K \approx\{1 / 2,5 / 2,7 / 2,11 / 2\}$ is quite clear. In conclusion, from both POD and DMD, in the full-domain asymmetric case additional dynamics is clearly observed compared to the symmetric configuration. 

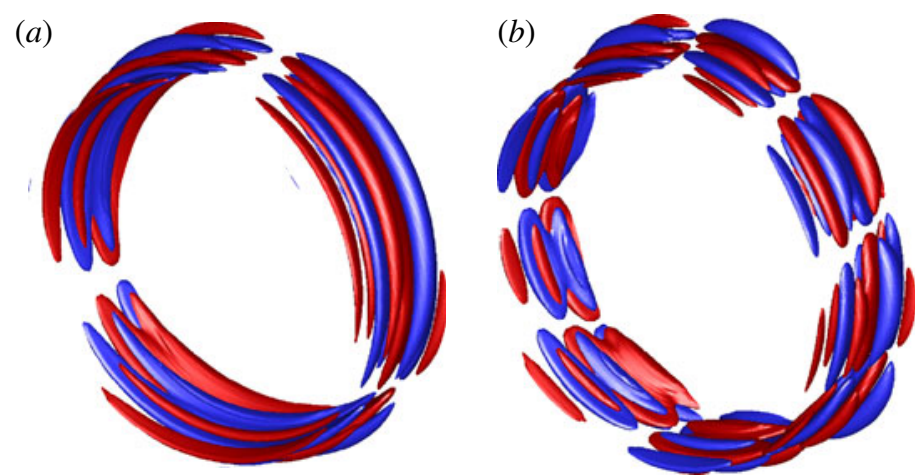

FIgURE 11. Dominant POD modes of symmetry case, one-third domain (case B in table 1): $(a)$ first group $K \approx 3 / 2, \overline{S t} \approx 1.85$, POD modes $2-3,(b)$ second group $K \approx 9 / 2$, $\overline{S t} \approx 5$, POD modes $4-5$. Here, $\overline{S t}$ denotes the average frequency content of the POD modes.
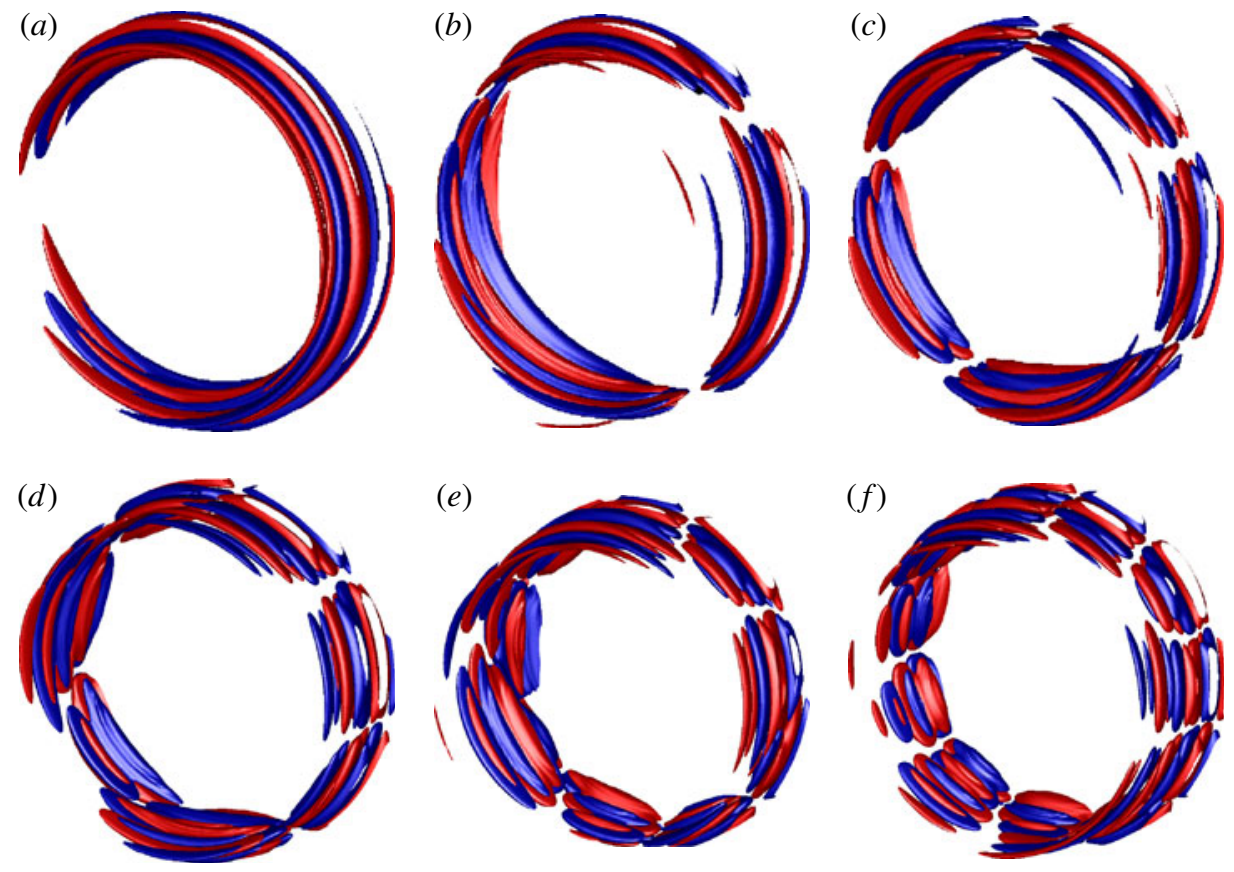

FIGURE 12. Dominant POD modes of asymmetry configuration (case E table 1): (a) first group $K \approx 1 / 2, \overline{S t} \approx 0.5$, POD modes $2-3,(b)$ second group $K \approx 3 / 2, \overline{S t} \approx 2.1$, POD modes $4-5,(c)$ third group $K \approx 5 / 2, \overline{S t} \approx 3$, POD modes $6-7,(d)$ fourth group $K \approx 7 / 2$, $\overline{S t} \approx 3.9$, POD modes $8-9$, (e) fifth group $K \approx 9 / 2, \overline{S t} \approx 4.9$, POD modes $13-14$ and $(f)$ sixth group $K \approx 11 / 2, \overline{S t} \approx 6.2$, POD modes $19-20$.

\section{Analysis of the modal decompositions}

It is well established that the presence of unstable modes in the wind turbine wake is related to the mutual inductance (vortex pairing) instability where there is an out-ofphase relationship between the waves on consecutive spirals, see e.g. Leishman et al. 
(a)

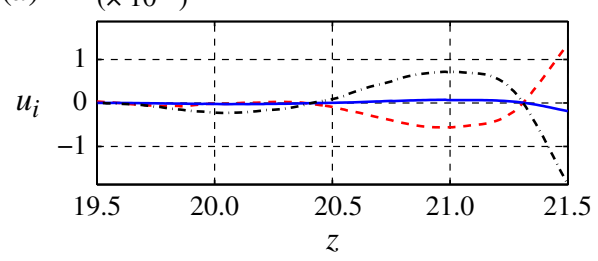

(b)

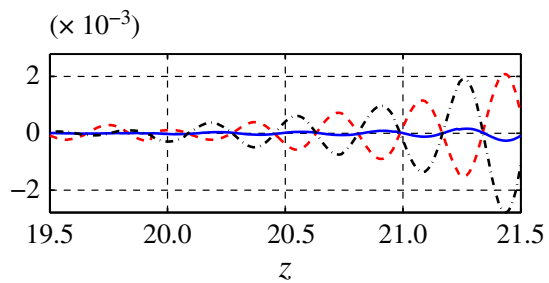

(c) $\quad\left(\times 10^{-3}\right)$

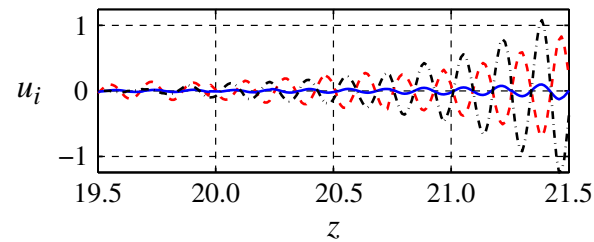

FIGURE 13 . Amplitude of the velocity components of asymmetric modes, case E: $(a)$ St $\approx$ $0.5(K \approx 0.5),(b) S t \approx 2(K \approx 1.5)$ and $(c) S t \approx 5(K \approx 4.5)$. The solid, dashed, dashed-dot lines represent the tangential, radial and axial velocity components, respectively.

(2004) and Ivanell et al. (2010). In other words, when we move out from one spiral to another, due to this oscillation the spirals move towards each other and can interact accordingly. This behaviour can be demonstrated if we analyse the disturbance velocity components on the dominant modes. In particular, we can observed a $180^{\circ}$ phase shift between the radial and axial components of the velocities (figure 13). These two components constitute the main component of the fluctuation velocity. This velocity implies that when we follow a fluid element along a spiral, as it moves in the positive radial direction, it stretches in the negative axial direction and vice versa. Therefore the two spirals depict an out-of-phase behaviour where they move closer and separate from each other simultaneously.

\subsection{Spatial analysis based on the dynamic modes}

A closer look at the velocity components of the dynamic modes indicates the presence of a wave-like motion, propagating along the spirals. DMD extracts dynamic modes corresponding to specific frequencies from which we can extract the growth rates pertaining to the spatial instability. In particular, if we follow the point with maximum energy along the spiral, we obtain the spatial growth as we move along the trajectory; the maximum energy along a spiral trajectory is determined by

$$
w(z)=\max \sqrt{u_{r}^{2}+u_{\theta}^{2}+u_{z}^{2}}, \quad r, \theta, z \in \text { spiral trajectory }
$$

where $u_{r}, u_{\theta}$ and $u_{z}$ are the real value of the radial, azimuthal and axial velocities of the modes. If we then assume an underlying exponential amplification, the spatial growth rate (denoted as $\tilde{\sigma}$ ) is defined as the slope of the maximum energy curve in logarithmic scale $\tilde{\sigma}=\mathrm{d}\left(\log \left(w / U_{c}\right)\right) / \mathrm{d} z$, with a reference speed $U_{c}$, here taken as the convection speed of the vortices in the axial direction. Subsequently, the non-dimensional spatial growth rate can be obtained, $\sigma=\tilde{\sigma}\left(2 h^{2} U_{c} / \Gamma\right)$, using the velocity of the vortex $\Gamma / 2 h$, the separation distance $h$ between successive helix loops (with $h$ being defined as the helix pitch divided by the number of blades) and the 

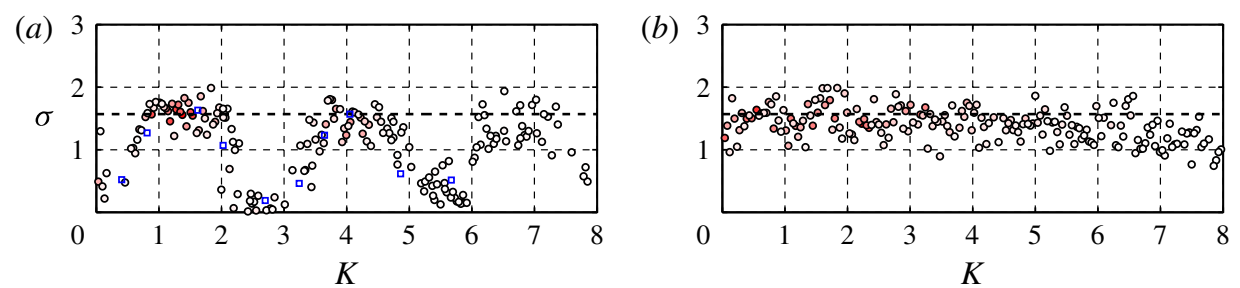

FIGURE 14. Spatial growth rate as function of wavenumber: $(a)$ symmetric, one-third domain (case B in table 1) and (b) asymmetric configuration (case E). Circles correspond to the modes of dynamic mode analysis and they are colored based on their DMD amplitudes from red (high amplitude) to white (low amplitude). The blue squares are the result of the one-third domain using harmonic excitation, case A (Ivanell et al. 2010). The central dashed lines correspond to $\sigma=\pi / 2$.

circulation $\Gamma$. In figure 14, the non-dimensional spatial growth rates for the first 300 energetic leading modes and for both configurations are depicted. Figure 14(a) shows clustering of the spatial growth rates for the symmetric configuration (case B). The growth rates follow a sinusoidal type of variation, where the most energetic DMD modes are the most spatially unstable ones. This behaviour is also reported by Leishman et al. (2004) and Ivanell et al. (2010) where the wavenumbers corresponding to the maximum growth rates occur at half-integer multiples of the number of the blades, i.e. $K=3(i+(1 / 2))$ for all integers $i$. Similarly, the minimum spatial growth rates occur at wavenumbers equal to integer multiples of the number of the blades. This result cannot be confirmed for the asymmetric setup (figure $14 b$ ). In this case all the modes including the ones observed in the symmetric case $(K=3 / 2$ and 9/2) have positive growth rates with a decreasing trend as we move towards higher frequencies. This trend implies that the deviation of the spiral from its helical path is limited at higher wavenumbers. The identification of the modes with the highest growth rate is important since these modes, if triggered, shorten the stable wake length behind the rotor; the stable wake length is defined as the distance between the rotor and the region where the wake breaks down. The present results suggest that the highest growth rate is around $\sigma \approx \pi / 2$ as previously found by Ivanell et al. (2010) if the spatial growth rates found in that study are properly rescaled. It is interesting to compare this to the temporal growth rates obtained by Lamb (1932) where the maximum possible temporal growth rate of helical vortices is shown to be $\pi / 2$ using a simple model. Other analytical and experimental investigations have also confirmed the same value (Levy \& Forsdyke 1927; Widnall 1972; Leweke et al. 2013). The fact that both the temporal and spatial growth rates are $\pi / 2$ indicates that the propagation velocity of the waves is unity. Note that the full-domain $120^{\circ}$ symmetric run (case D) features modes with growth rates slightly above $\pi / 2$ (mainly for weaker modes, not shown here). However, we believe that this is a consequence of the larger sensitivity due to potential subharmonic excitation in this case.

To further illuminate the discussion, we remark that the unstable wavenumbers with a maximum growth rate occur when the waves on two consecutive spirals are completely out-of-phase and a stable configuration with a minimum (zero) growth rate is observed when they are in-phase (Leishman et al. 2004; Ivanell et al. 2010). In figure 15, we present a sketch indicating certain wavenumbers for unrolled tip spirals and a three-bladed rotor. For instance, for the wavenumbers equal to half-multiples of odd integer numbers, $K=i / 2$, for all odd integers $i=1,3, \ldots$, the out-of-phase 


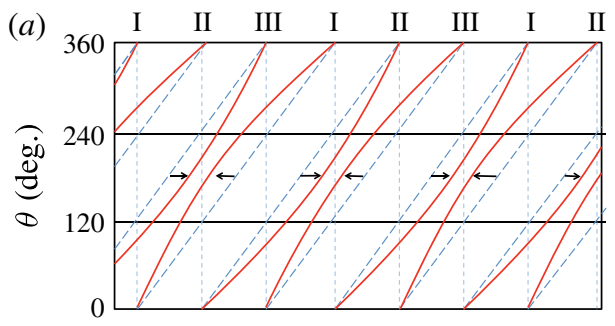

(b)
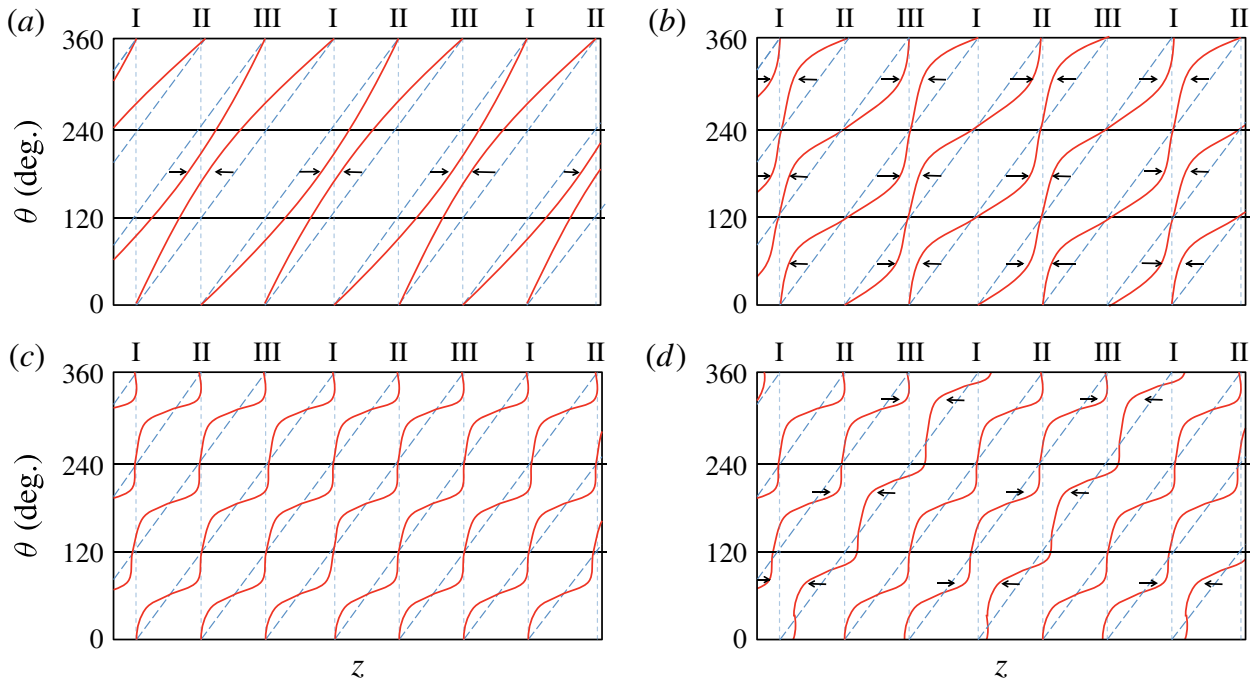

(d)
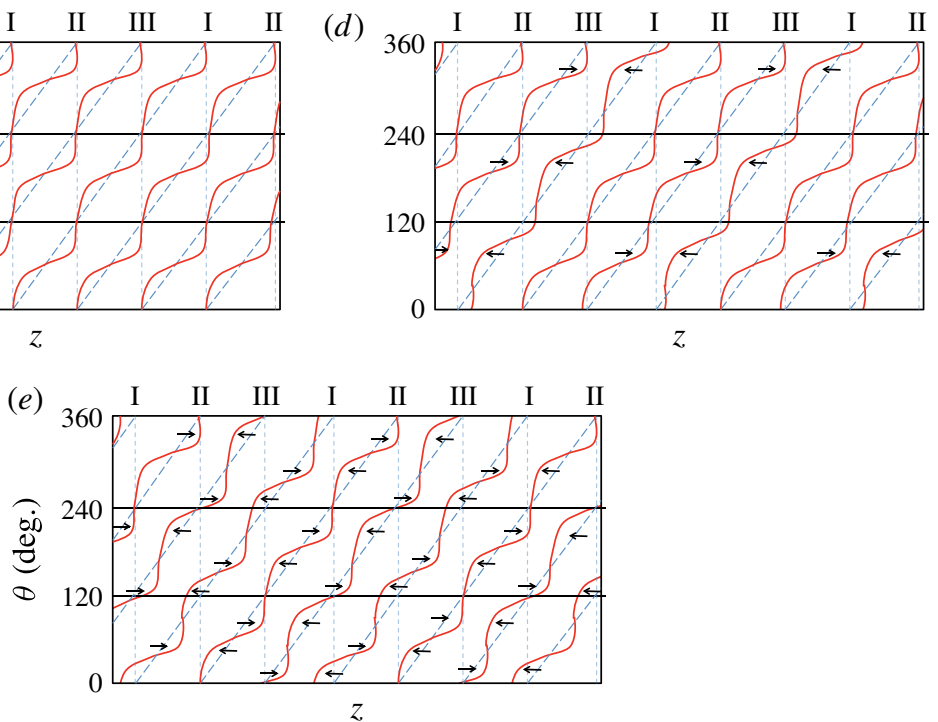

FIGURE 15. A schematic depiction of the unrolled tip spirals in which the mutual interaction between the subsequent vortices can occur. The inclined dashed and solid lines represent the unperturbed and perturbed tip spirals, respectively. The arrows show the position of the local vortex pairing. (a) $K \approx 0.5$, (b) $K \approx 3 / 2$, (c) $K \approx 3$ : three in-phase spirals (stable configuration). $(d) K \approx 3$, two in-phase spiral with one random phase (unstable configuration) and $(e) K \approx 3$, three random-phase spirals (unstable configuration). Maximum displacement occurs at $2 K$ locations for each revolution triggering local pairing.

relationship can be easily confirmed. The perturbation wavenumbers corresponding to $K=1 / 2$ and $K=3 / 2$ are depicted in figure $15(a, b)$ where an out-of-phase behaviour is clearly observed between two consecutive spirals. This behaviour consequently results in a local formation of vortex pairing and hence leads to an unstable configuration. Now, consider the same phenomenon for wavenumbers equal to half-multiples of even numbers $K=i / 2$, for all even integers $i=2,4, \ldots$. In particular, we focus our attention on wavenumber $K=3$ for different configurations. In the $120^{\circ}$ symmetric configuration, where we impose a periodicity on one-third of the domain, we have a constraint on the phase of the waves at every $120^{\circ}$ of the domain. In other words, the phase of the waves for all the blades has to be the same at this point. Hence, the out-of-phase relationship can only be identified for the wavenumbers equal to half-multiples of odd integer numbers $K=3 i / 2$, for all odd integers $i$. Similarly, the in-phase relationship occurs at half-multiples of even integer numbers $K=3 i / 2$, for all odd integers. In fact, due to the phase restriction some unstable wavenumbers, identified previously in the asymmetric setup are now completely 
eliminated; e.g. $K=1 / 2$. Furthermore, the wavenumber $K=3$ leads to a stable configuration with an in-phase relationship between the waves (figure 15c). However, if we remove the phase constraint and consider the asymmetric configuration, we can adjust an appropriate phase on blade III to be out-of-phase with blade I while it is still in phase with blade II (figure 15d). Subsequently, we can adjust the phase on blade II to have a completely out-of-phase relationship between each consecutive spiral (figure 15e). Hence the wavenumber $K=3$ which was found to be originally stable in the symmetric setup leads to an unstable configuration when the complete $360^{\circ}$ case without symmetry conditions is considered.

These observations imply that the results of the previous investigations by Leishman et al. (2004) and Ivanell et al. (2010) most likely were based on a phase-restricted system and thus not applicable to the full $360^{\circ}$ setup. In fact, with the same reasoning, we can conclude that all the wavenumbers in the asymmetric configuration can lead to an unstable configuration provided that the waves have appropriate phases, which would be the case for completely random excitation e.g. due to free-stream turbulence.

\section{Model for determining the stable wake length}

By defining the length of the near wake behind a rotor, $l$, as the axial distance from the rotor plane to the position where the tip vortices break down into small-scale turbulence, it is possible to formulate an analytical relationship between this length and the operating conditions of the rotor using linear stability theory. From linear stability theory assuming exponential growth we obtain that the amplitude amplification is given as

$$
\frac{A}{A_{0}}=\mathrm{e}^{\tilde{\sigma} z},
$$

where $A_{0}$ is the initial perturbation at location $z=z_{0}, A$ is the amplitude at position $z$, and $\tilde{\sigma}$ is the dimensional growth rate of the pairing instability. Assuming that the vortices propagate downstream with a constant velocity $U_{c}$, one can establish a relation between spatial and temporal growth according to

$$
A(t)=A_{0} \mathrm{e}^{\tilde{\sigma} U_{c} t},
$$

where $t$ denotes the time, $t=U_{c} / z$. As discussed in $\S 5$ the non-dimensional spatial growth rate is given by the universal expression

$$
\sigma=\tilde{\sigma} \frac{2 h^{2} U_{c}}{\Gamma}=\frac{\pi}{2},
$$

hence

$$
\tilde{\sigma}=\frac{\pi \Gamma}{4 h^{2} U_{c}} .
$$

This expression is related only to the parameters of the vortices in the wake, e.g. their strength and mutual distance. What is required, however, are the parameters associated with the operational conditions of the wind turbine rotor. In order to establish these relations, we exploit some basic results from momentum theory. Assuming that the wake essentially consists of a system of tip vortices of strength $\Gamma$ and a root vortex strength of $-N_{b} \Gamma$, where $N_{b}$ denotes the number of blades, the following geometrical relationship can be established:

$$
\frac{N_{b} h}{2 \pi R}=\frac{U_{c}}{\Omega R},
$$


where $\Omega R$ is the tip speed of the rotor. From this we thus get

$$
h=\frac{2 \pi R \widetilde{U}_{c}}{N_{b} \lambda},
$$

where $\lambda$ is the tip speed ratio and $\widetilde{U}_{c}=U_{c} / U_{0}$. Assuming that the rotor is loaded with a constant circulation and neglecting the influence of nonlinear rotational terms, we obtain the following approximate expression for the thrust: (see Sørensen \& van Kuik 2011),

$$
T=\frac{1}{2} \rho R^{2} \Omega N_{b} \Gamma
$$

Introducing further the thrust coefficient,

$$
C_{T}=\frac{T}{(1 / 2) \rho \pi R^{2} U_{0}^{2}},
$$

we get

$$
\Gamma=\frac{\pi U_{0}^{2} C_{T}}{\Omega N_{b}} .
$$

Inserting (6.6) and (6.9) into (6.4), we get

$$
\sigma=\frac{N_{b} C_{T} \lambda}{16 \widetilde{U}_{c}^{3} R}
$$

Further, combining (6.10) with (6.1) results in

$$
A(z)=A_{0} \exp \left[\frac{N_{b} C_{T} \lambda}{16 \widetilde{U}_{c}^{3}}\left(\frac{z}{R}\right)\right] .
$$

Rearranging this equation results in

$$
\left(\frac{z}{R}\right)=\frac{16 \widetilde{U}_{c}^{3}}{N_{b} \lambda C_{T}} \log \left(\frac{A(z)}{A_{0}}\right)
$$

From the previous work by Ivanell et al. (2010) it was found that the nonlinear breakdown process starts when the amplitude amplification reaches the ratio between original perturbation and the undisturbed wind velocity, i.e. when

$$
\frac{A_{\max }(t)}{A_{0}} \approx \frac{U}{u^{\prime}} .
$$

Furthermore, assuming that the turbulence intensity, $T_{i}$, is proportional to the perturbation, we get

$$
\frac{u^{\prime}}{U}=C_{1} T_{i} .
$$

Assuming that the turbulence intensity is equal to a constant $C_{1}$ times the perturbation amplitude, and by inserting (6.14) into (6.12):

$$
\left(\frac{l}{R}\right)=\frac{-16 \widetilde{U}_{c}^{3}}{N_{b} \lambda C_{T}} \log \left(C_{1} T_{i}\right) .
$$


To the first approximation the propagation velocity of the vortices is given by the free-stream velocity, i.e. $U_{c}=U_{0}$, and we have arrived at the final expression for the wake length in terms of wind turbine properties. A more accurate propagation velocity maybe found by exploiting the so-called roller-bearing analogy, in which it is assumed that the vortices move with the average velocity between the wake velocity and the undisturbed wind speed, $U_{0}$ (see e.g. Okulov \& Sørensen 2007). However, it may turn out that the convection velocity is somewhat higher than the one indicated by the roller-bearing analogy and lower than the free-stream velocity. We therefore introduce the following expression for the convection velocity:

$$
U_{c}=C_{2} U_{w}+\left(1-C_{2}\right) U_{0},
$$

where $U_{w}$ is the wake velocity and $C_{2} \in[0,1]$ is a constant that needs to be calibrated against measurements. Using the axial momentum theory $U_{w}=U_{0} \sqrt{1-C_{T}}$, we get

$$
\widetilde{U}_{c}=1+C_{2}\left[\sqrt{1-C_{T}}-1\right] .
$$

Introducing this into (6.15), we arrive at the final expression for the length of the near wake

$$
\left(\frac{l}{R}\right)=\frac{-16\left[1+C_{2}\left(\sqrt{1-C_{T}}-1\right)\right]^{3}}{N_{b} \lambda C_{T}} \log \left(C_{1} T_{i}\right) .
$$

This expression gives a measure of the length of the near wake as a function of the intensity of the ambient turbulence level, $T_{i}$, and of parameters depending uniquely on the turbine's operational characteristics. The two unknown parameters $\left(C_{1}\right.$ and $C_{2}$ ) should be calibrated using wind turbine data either from experiments or from simulations.

\section{Conclusions}

To determine the stability properties of wind turbine wakes, a numerical study has been carried out based on the Tjæreborg wind turbine. The numerical model is based on LES of the Navier-Stokes equations using the ACL method. The wake is perturbed by applying stochastic or harmonic excitations in the neighbourhood of the tips of the blades. The flow fields are then analysed using two modal decomposition techniques, DMD and POD. The study focuses on the stability of the tip vortices on the wind turbine wakes. According to the grid configuration and the excitation type, we studied two symmetric and asymmetric conditions: two cases where a $120^{\circ}$ symmetry is imposed either by confining the mesh to only $120^{\circ}$ or by imposing the same disturbances in front of each blade retaining the complete $360^{\circ}$ setup. Also, a completely asymmetric case using uncorrelated disturbances for each blade has been used as well.

We found that the amplification of specific waves (travelling structures) along the spiral is responsible for triggering the instability leading to wake breakdown. The presence of unstable modes in the wake is related to the mutual inductance (vortex pairing) instability where there is an out-of-phase relationship between the waves on consecutive spirals. These dominant structures can be categorized by their modal shape and frequency content. Two groups of modes are identified for the symmetric configurations and four extra groups in addition to the symmetric modes are obtained for the asymmetry condition. These extra modes can be explained through the fact 
that phase restriction between the spirals in the correlated setup limits the modes leading to instability. The modal decomposition analysis thus shows that a one-third domain with periodic boundary conditions has similar dynamics to a full domain if identical perturbations are imposed. However the full geometry, if disturbed truly randomly, does not necessarily produce the same results as the symmetric modes. These new modes, which can only be seen if the full-domain asymmetric case is considered, are involved in the process of tip vortex instability. Further analysis of the dominant dynamic modes reveals an out-of-phase behaviour between the radial and axial velocities on the tip vortices implying that the main cause of wake destabilization is the mutual inductance instability.

Using the non-dimensional growth rate, we found that the pairing instability has a universal growth rate equal to $\pi / 2$. Using this relationship, and the assumption that breakdown to turbulence occurs once a vortex has experienced sufficient growth, we provide an analytical relationship between the turbulence intensity and the stable wake length.

\section{Acknowledgements}

The work has been carried out with the support of the Danish Council for Strategic Research for the project Center for Computational Wind Turbine Aerodynamics and Atmospheric Turbulence (grant 2104-09-067216/DSF) (COMWIND) and the Nordic Consortium on Optimization and Control of Wind Farms. Computer time on Lindgren was granted by the Swedish National Infrastructure for Computing (SNIC). PDC Center for High-Performance Computing is acknowledged for providing the post-processing facility Ellen.

The authors would like to acknowledge $\operatorname{Dr} \mathrm{T}$. Leweke for fruitful discussions regarding the pairing instability mechanism.

\section{Appendix. Modal decompositions}

\section{A.1. Proper orthogonal decomposition}

POD is a well-known procedure to capture the coherent structures of a flow. This method extracts an orthogonal basis for the decomposition of a given collection of snapshots. POD has its roots in statistical analysis and has appeared with various names, including: empirical eigenfunctions, Karhunen-Loève decomposition or empirical orthogonal functions. POD was first introduced in turbulence by Lumley (1970) and the method has been described in detail in many publications (see e.g. Aubry 1991; Manhart \& Wengle 1993). The benefit of using POD is its property of fast convergence to get an accurate reconstruction with a low number of modes as opposed to, for example, Fourier decomposition (Frederich \& Luchtenburg 2011). On the other hand, since POD extracts an orthogonal basis ranked by energy content, it is not always straightforward to interpret the physical meaning of the spatial modes and their temporal counterparts. In particular, in complex flows, different spatial structures and temporal scales are often present in one mode.

The derivation of POD begins with collecting a set of $m$ snapshots $\boldsymbol{u}_{j}, \quad j=$ $0, \ldots, m-1$ that lie in a vector space $\boldsymbol{u}_{j} \in \mathbb{U} \subset \mathbb{R}^{n}$ with a corresponding inner product defined as

$$
\left\langle\boldsymbol{u}_{i}, \boldsymbol{u}_{j}\right\rangle_{M}=\boldsymbol{u}_{i}^{\mathrm{T}} \boldsymbol{M} \boldsymbol{u}_{j} \quad \forall \boldsymbol{u}_{i}, \boldsymbol{u}_{j} \in \mathbb{U}, \quad \text { and } \quad\left\|\boldsymbol{u}_{j}\right\|_{M}^{2}=\left\langle\boldsymbol{u}_{j}, \boldsymbol{u}_{j}\right\rangle_{M},
$$

where the symbol ${ }^{\mathrm{T}}$ denotes the transpose and $\boldsymbol{M} \in \mathbb{R}^{n \times n}$ represents the diagonal spatial weight matrix in which the entries (weights) are for instance given by the 
local cell volumes corresponding to each grid point. The aim of the POD is to find a set of deterministic and orthogonal basis functions, $\boldsymbol{X}$, and their corresponding time coefficients $\boldsymbol{T}$ to decompose the snapshots

$$
\boldsymbol{U}_{m}=\boldsymbol{X} \boldsymbol{T}=\boldsymbol{X} \boldsymbol{\Sigma} \boldsymbol{W}^{\mathrm{T}},
$$

where $\|\boldsymbol{X}\|_{M}=\boldsymbol{I},\|\boldsymbol{W}\|_{N}=\boldsymbol{I}, \boldsymbol{N} \in \mathbb{R}^{n \times n}$ represent the diagonal temporal weight matrix and $\boldsymbol{I}$ is the identity matrix.

To obtain the decomposition (A 2), we first compute the singular value decomposition (SVD) of $\boldsymbol{M}^{1 / 2} \boldsymbol{U}_{m} \boldsymbol{N}^{1 / 2}=\hat{\boldsymbol{X}} \boldsymbol{\Sigma} \hat{\boldsymbol{W}}^{\mathrm{T}}$ where $\hat{\boldsymbol{X}}$ and $\hat{\boldsymbol{W}}$ are unitary matrices: $\hat{\boldsymbol{X}}^{\mathrm{T}} \hat{\boldsymbol{X}}=\boldsymbol{I}$ and $\hat{\boldsymbol{W}}^{\mathrm{T}} \hat{\boldsymbol{W}}=\boldsymbol{I}$. Then the POD modes are obtained as $\boldsymbol{X}=\boldsymbol{M}^{-1 / 2} \hat{\boldsymbol{X}}$ and $\boldsymbol{W}=\boldsymbol{N}^{-1 / 2} \hat{\boldsymbol{W}}$.

\section{A.2. Dynamic mode decomposition}

Unlike POD, in DMD for each mode a specific temporal frequency is assigned. Therefore, the physical interpretation of the various modes as dynamic components of the complete flow might be easier. We base the following description on Rowley et al. (2009) and the subsequent more stable implementation put forward by Schmid (2010). To compute DMD, we consider a sufficiently long, but finite time series of snapshots. We assume a linear mapping that associates the flow field $\boldsymbol{u}_{j}$ to the subsequent flow field $\boldsymbol{u}_{j+1}$ such that

$$
\boldsymbol{u}\left(\boldsymbol{x}_{i}, t_{j+1}\right)=\boldsymbol{u}_{j+1}=\mathrm{e}^{\tilde{A} \Delta t} \boldsymbol{u}_{j}=\boldsymbol{A} \boldsymbol{u}_{j} .
$$

Hence, it is possible to write

$$
\boldsymbol{u}\left(\boldsymbol{x}_{i}, t_{j}\right)=\sum_{k=0}^{m-1} \phi_{k}\left(\boldsymbol{x}_{i}\right) \boldsymbol{a}_{k}\left(t_{j}\right)=\sum_{k=0}^{m-1} \phi_{k}\left(\boldsymbol{x}_{i}\right) \mathrm{e}^{\mathrm{i} \omega_{k} j \Delta t}=\sum_{k=0}^{m-1} \phi_{k}\left(\boldsymbol{x}_{i}\right) \lambda_{k}^{j} .
$$

In this equation, $\mathrm{i} \omega_{k}$ and $\lambda_{k}$ are the eigenvalues of the matrices $\tilde{\boldsymbol{A}}$ and $\boldsymbol{A}$, respectively, and the spatial modes are collected in the eigenfunctions $\phi_{k}$. The complex frequency containing both growth rate and physical oscillation circular frequency are related to the eigenvalue through $\lambda_{k}=\mathrm{e}^{\mathrm{i} \omega_{k} \Delta t}$. In order to define a unique amplitude for each DMD mode, we split $\phi_{k}=\boldsymbol{v}_{k} d_{k}$ where $\left\|\boldsymbol{v}_{k}\right\|_{M}=1$. We may then define $d_{k}$ as the amplitude and $d_{k}^{2}$ as the energy of the dynamic mode $\phi_{k}$; the $\lambda_{k}$ are associated with the time development of the spatial expansion modes $\boldsymbol{v}_{k}$. Such an expansion can be interpreted as a finite-sum version of the Koopman expansion where the Koopman eigenvalues, $\lambda_{k}$, dictate the growth rate and frequency of each mode (Rowley et al. 2009). Without pursuing this further, it is sufficient to state that the Koopman expansion puts the DMD on a firm theoretical foundation.

For the practical implementation of the DMD, we start with (A4) and rewrite it in matrix form as

$$
\begin{aligned}
\boldsymbol{U}_{m} & =\left[\boldsymbol{v}_{0}, \boldsymbol{v}_{1}, \ldots, \boldsymbol{v}_{m-1}\right]\left(\begin{array}{cccc}
d_{0} & 0 & \cdots & 0 \\
0 & d_{1} & \cdots & 0 \\
\vdots & \vdots & \ddots & \vdots \\
0 & 0 & \cdots & d_{m-1}
\end{array}\right)\left(\begin{array}{ccccc}
1 & \lambda_{0} & \lambda_{0}^{2} & \cdots & \lambda_{0}^{m-1} \\
1 & \lambda_{1} & \lambda_{1}^{2} & \cdots & \lambda_{1}^{m-1} \\
\vdots & \vdots & \vdots & \ddots & \vdots \\
1 & \lambda_{m-1} & \lambda_{m-1}^{2} & \cdots & \lambda_{m-1}^{m-1}
\end{array}\right) \\
& =\tilde{\boldsymbol{X}} \boldsymbol{D} \boldsymbol{S}=\overline{\boldsymbol{X}} \boldsymbol{S}
\end{aligned}
$$


where $\boldsymbol{S}$ is a so-called Vandermonde matrix and dictates the time evolution of the dynamic modes. Note that the normalized dynamic modes $\tilde{\boldsymbol{X}}$ are scaled by their corresponding amplitudes in $\overline{\boldsymbol{X}}$.

To compute the DMD modes $\overline{\boldsymbol{X}}$, we proceed as follows. As the number of snapshots increases, it is reasonable to assume that beyond a certain limit, the snapshot matrix becomes linearly dependent. In other words, adding additional flow fields $\boldsymbol{u}_{j}$ to the data set will not improve the rank of snapshot matrix $\boldsymbol{U}_{m}$. Hence, one can obtain the flow field sequence $\boldsymbol{U}_{m+1}=\left[\boldsymbol{u}_{1}, \ldots, \boldsymbol{u}_{m}\right]$ by a linear combination of the previous snapshot sequence $\boldsymbol{U}_{m}$. This step is expressed as

$$
\boldsymbol{U}_{m+1}=\boldsymbol{U}_{m} \boldsymbol{C}+\epsilon \boldsymbol{e}^{\mathrm{T}},
$$

where $\boldsymbol{e}=[0, \ldots, 0,1]^{\mathrm{T}}$ and $\epsilon$ contains the residual. This procedure will result in the low-dimensional system matrix $\boldsymbol{C}$ which is of companion-matrix type and can be computed using a least-square technique (Rowley et al. 2009). The matrix $\boldsymbol{C}$ can be diagonalized as

$$
\boldsymbol{C}=\boldsymbol{S}^{-1} \boldsymbol{\Lambda} \boldsymbol{S}=\boldsymbol{Z} \boldsymbol{\Lambda} \boldsymbol{Z}^{-1}, \quad \boldsymbol{\Lambda}=\operatorname{diag}\left(\lambda_{1}, \ldots, \lambda_{m}\right),
$$

where $\boldsymbol{S}$ is the Vandermonde matrix and $\boldsymbol{Z}$ is the eigenvector matrix using proper scaling such so that

$$
\boldsymbol{Z}=\boldsymbol{S}^{-1} \boldsymbol{D}^{-1} \text {. }
$$

The (diagonal) amplitude matrix $\boldsymbol{D}$ will be derived later. The eigenvalues of $\boldsymbol{C}$, collected in $\boldsymbol{\Lambda}$, are also referred to as the Ritz values and approximate some of the eigenvalues of the full nonlinear system. The dynamic modes, $\overline{\boldsymbol{X}}$, are given by

$$
\overline{\boldsymbol{X}}=\boldsymbol{U}_{m} \boldsymbol{S}^{-1}=\boldsymbol{U}_{m} \boldsymbol{Z D}=\tilde{\boldsymbol{X}} \boldsymbol{D},
$$

where $\tilde{\boldsymbol{X}}=\boldsymbol{U}_{m} \boldsymbol{Z}$ contain the modal structures. This method is mathematically correct but practical implementations might yield a numerically ill-conditioned algorithm (Schmid 2010). This is especially the case when the data set is rather large and noise contaminated. An improvement of this algorithm was proposed by Schmid (2010), where a self-similar transformation of the companion matrix $\tilde{\boldsymbol{C}}$ is obtained as a result of the projection of the velocity fields on the subspace spanned by the corresponding POD modes. The preliminary step of the algorithm is to perform the POD of the velocity field sequence

$$
\boldsymbol{U}_{m}=\boldsymbol{X} \boldsymbol{\Sigma} \boldsymbol{W}^{\mathrm{T}} .
$$

Using this decomposition the self-similar transformation of the companion matrix $\tilde{\boldsymbol{C}}$ is defined as

$$
\tilde{\boldsymbol{c}}=\boldsymbol{\Sigma} \boldsymbol{W}^{\mathrm{T}} \boldsymbol{C} \boldsymbol{W} \boldsymbol{\Sigma}^{-1} .
$$

The transformed companion matrix $\tilde{\boldsymbol{C}}$, related to $\boldsymbol{C}$ via this transformation, is a full matrix which improves the conditioning of the eigenvalue problem. By computing an optimal companion matrix using (A 6) and (A 10) and re-arranging (A 11), we obtain

$$
\tilde{\boldsymbol{C}}=\boldsymbol{X}^{\mathrm{T}} \boldsymbol{M} \boldsymbol{U}_{m+1} \boldsymbol{W} \boldsymbol{\Sigma}^{-1}=\boldsymbol{Y} \boldsymbol{\Lambda} \boldsymbol{Y}^{-1},
$$

where $\boldsymbol{Y}=\left(\boldsymbol{y}_{1}, \ldots, \boldsymbol{y}_{\boldsymbol{m}}\right)$ is the eigenvector matrix with $\boldsymbol{y}_{k}^{\mathrm{T}} \boldsymbol{y}_{k}=1 \forall k=0, \ldots, m-1$. Moreover, the eigenvalues of $\tilde{\boldsymbol{C}}$ are the same as for $\boldsymbol{C}$, while their eigenvectors are related by

$$
\boldsymbol{Z}=\boldsymbol{W} \boldsymbol{\Sigma}^{-1} \boldsymbol{Y} .
$$


The modal structures $\tilde{\boldsymbol{X}}$ are extracted from the transformed companion matrix $\tilde{\boldsymbol{C}}$ in the following way,

$$
\tilde{\boldsymbol{X}}=\boldsymbol{U}_{m} \boldsymbol{Z}=\boldsymbol{U}_{m} \boldsymbol{W} \boldsymbol{\Sigma}^{-1} \boldsymbol{Y}=\boldsymbol{X} \boldsymbol{\Sigma} \boldsymbol{W}^{\mathrm{T}} \boldsymbol{W} \boldsymbol{\Sigma}^{-1} \boldsymbol{Y}=\boldsymbol{X} \boldsymbol{Y} .
$$

It is important to note that the norm $\|\cdot\|_{M}$ of the columns of $\tilde{\boldsymbol{X}}$ is unity. To obtain the amplitudes of the DMD modes without inverting the Vandernmode matrix $\boldsymbol{S}$, we benefit from the fact that $\boldsymbol{D}$ is a diagonal complex matrix. Combining (A 9), (A 10) and (A 14), we obtain

$$
\overline{\boldsymbol{X}}=\boldsymbol{U}_{m} \boldsymbol{S}^{-1}=\boldsymbol{X} \boldsymbol{\Sigma} \boldsymbol{W}^{\mathrm{T}} \boldsymbol{S}^{-1}=\tilde{\boldsymbol{X}} \boldsymbol{D}=\boldsymbol{X} \boldsymbol{Y} \boldsymbol{D} \Rightarrow \boldsymbol{D}^{-1}=\boldsymbol{S} \boldsymbol{W} \boldsymbol{\Sigma}^{-1} \boldsymbol{Y} .
$$

Here, $\boldsymbol{D}$ can now be computed by inverting the diagonal entries of matrix $\boldsymbol{D}^{-1}$. Note that (A 13) gives a correct scaling for the matrix $\boldsymbol{Z}$. By combining (A 13) and (A 15) one can indeed confirm that the original definition for matrix $\boldsymbol{Z}$, (A 8), is satisfied.

To compute the modal decompositions, we need to specify the sampling interval between two consecutive snapshots $\Delta t$ and the number of snapshots $m$. The choice of these parameters has a significant effect on the convergence of the modal decomposition. In particular, convergence is achieved if any variation in the values of these parameters does not 'significantly' change the results. The sampling interval $\Delta t$ should be adjusted according to the Nyquist criterion. According to this criterion, the maximum frequency that we can resolve is $f=1 /(2 \Delta t)$. In fact, this frequency depends on the highest physically relevant frequency in the flow. The number of snapshots, $m$, on the other hand, has to be chosen such that the total time spanned by the snapshots is long enough to capture the slowest scales of the flow. In other words, convergence is achieved if, by increasing the number of snapshots or decreasing the sampling interval, the resulting modes and their corresponding amplitudes are not changed. In this paper, the mean of the snapshots is subtracted from the data set. Hence the results are equivalent to the temporal Fourier transform as showed by Chen et al. (2012). Furthermore, to estimate the power spectral density a windowing/Welch type of method is implemented.

\section{A.3. Dealing with noisy data sets}

We compute the energy of the modes as a function of frequency (power spectral density). An estimate of the power density is referred to as a periodogram. In our setup, cases B, D and E, we excited the instabilities with random white noise in order to trigger all possible frequencies of the system. However, a number of difficulties arises when we are dealing with a noisy data set. The raw periodogram (without any smoothing) is not a good spectral estimate as it suffers from side-lobe energy leakage (bias) in a frequency band where the true spectrum is zero (Rao, Hamed \& Chen 2003). Another problem is that the variance of the energy at a given frequency does not decrease as the number of samples increases. The former problem arises since we have a limited number of samples and a sharp truncation exists at the end of the data set. A remedy is to multiply the samples by a window function which truncates the sequence gradually rather than abruptly. The latter problem (variance) can be improved by smoothing the periodogram. Such a technique is known as the Welch method (Welch 1967). The Welch method reduces the variance in the periodogram in exchange for reducing the frequency resolution caused by averaging used in the algorithm. The computational procedure is to split the data into several overlapping segments, compute the power spectral density of each segment, and average the resulting periodograms to produce the final estimate of the power spectral density. 
(a)

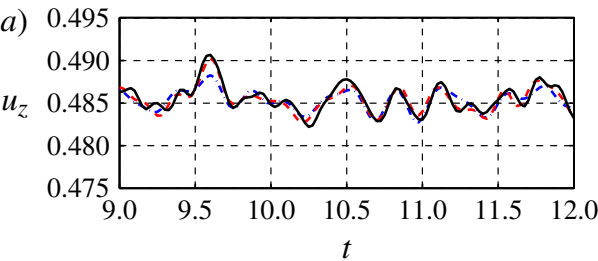

(b)

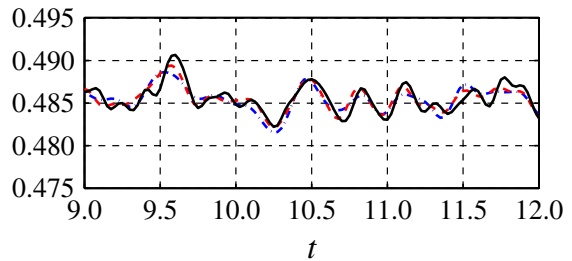

(c)

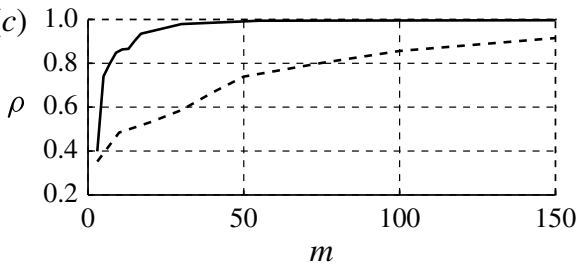

FIgURE 16. (Colour online) Time evolution of the axial velocity component recorded near the vortex centre of the asymmetric configuration (case E). The probe is located at $z=21$ and $r=1.25$. The black line represents the original signal. (a) Reconstruction of the signal using different numbers of POD modes: the first leading nine leading modes (dashed) and 17 leading modes (dashed-dot line). (b) Reconstruction of the signal using the leading 100 (dashed line) and 150 (dashed-dot line) dominant DMD modes. (c) Correlation coefficients between the signal of the probe and the reconstructed signal using different numbers of DMD (solid line) and POD modes (dashed line).

\section{A.4. Low-order model of wind turbine wake}

Based on the modal decomposition of the flow, it is possible to introduce a filtering technique that removes presumably less dynamically important structures from the flow, thus yielding a low-order model of the wind turbine wake. A superposition of sufficiently many dominant modes would capture the most important large-scale dynamics and could reproduce the main features of the original snapshot sequence. In figure $16(a, b)$ we investigate the required number of modes to reconstruct a signal near the vortex centre of the asymmetric full-domain configuration (case E). In fact, the fluctuation velocities near the vortex centre are small compared to the mean flow.

Figure 16(c) shows the correlation coefficient, $\rho$, between the original signal and the reconstructed one. The correlation coefficients is a number between $-1 \leqslant \rho \leqslant 1$; the upper bound $\rho=1$ indicates that the two signals are the same while the lower bound $\rho=-1$, suggests that the two signals are anti-correlated. Figure 16(c) implies that a smaller number of POD modes is necessary to capture the same dynamics compared to DMD. It is also observed that the reconstructed flow field in both cases captures the dynamic of the large scales and filters out the higher frequencies from the system in a global way.

\section{REFERENCES}

Alfredsson, P. H. \& Dahlberg, J. A. 1979 A preliminary wind tunnel study of windmill wake dispersion in various flow conditions. Tech. Note AU-1499, Part 7.

AUbry, N. 1991 On the hidden beauty of the proper orthogonal decomposition. Theor. Comput. Fluid Dyn. 2 (5), 339-352.

Bhagwat, M. J. \& Leishman, J. G. 2001 Stability, consistency and convergence of numerical algorithms for time-marching free-vortex wake analysis. J. Am. Helicopter Soc. 46 (1), 59-70. 
Chen, K. K., Tu, J. H. \& Rowley, C. W. 2012 Variants of dynamic mode decomposition: boundary condition, Koopman, and Fourier analyses. J. Nonlinear Sci. 22 (6), 887-915.

Chevalier, M., Schlatter, P., Lundbladh, A. \& Henningson, D. S. 2007 SiMSON: a pseudospectral solver for incompressible boundary layer flows. Tech. Rep. TRITA-MEK 2007:07. Royal Institute of Technology, Stockholm, Sweden.

Felli, M., CAmussi, R. \& Di Felice, F. 2011 Mechanisms of evolution of the propeller wake in the transition and far fields. J. Fluid Mech. 682, 5-53.

Frederich, O. \& Luchtenburg, D. M. 2011 Modal analysis of complex turbulent flow. In 7th Intl Symp. on Turbulence and Shear Flow Phenomena (TSFP-7), Ottawa, Canada.

Gupta, B. P. \& Loewy, R. G. 1974 Theoretical analysis of the aerodynamic stability of multiple, interdigitated helical vortices. AIAA J. 12 (10), 1381-1387.

IVAnell, S., Mikkelsen, R., Sørensen, J. N. \& Henningson, D. S. 2010 Stability analysis of the tip vortices of a wind turbine. Wind Energy 13 (8), 705-715.

Jain, R., Conlisk, A. T., Mahalingam, R. \& Komerath, N. M. 1998 Interaction of tip-vortices in the wake of a two-bladed rotor. In 54th Annual Forum of the Am. Helicopter Soc., Washington, $D C$.

JoukowsKi, N. E. 1912 Vortex theory of screw propeller. Trudy Otdeleniya Fizicheskikh Nauk Obshchestva Lubitelei Estestvoznaniya 16 (1), 1-31.

Lamb, H. 1932 Hydrodynamics, 6th edn Cambridge University Press.

Leishman, G., Bhagwat, M. J. \& Ananthan, S. 2004 The vortex ring state as a spatially and temporally developing wake instability. J. Am. Helicopter Soc. 49 (2), 160-175.

LEVY, H. \& FORSDYKE, A. G. 1927 The stability of an infinite system of circular vortices. Proceedings R. Soc. Lond. A 114 (768), 594-604.

LEVy, H. \& Forsdyke, A. G. 1928 The steady motion and stability of a helical vortex. Proc. R. Soc. Lond. A 120 (786), 670-690.

Leweke, T., Bolnot, H., Quaranta, U. \& Dizès, S. Le. 2013 Local and global pairing in helical vortex systems. Intl Conf. on Aerodynamics of Offshore Wind Energy Systems and Wakes, Lyngby, Denmark.

Lumley, J. L. 1970 Stochastic Tools in Turbulence. Academic.

MANhaRT, M. \& Wengle, H. 1993 A spatiotemporal decomposition of a fully inhomogeneous turbulent flow field. Theor. Comput. Fluid Dyn. 5 (4), 223-242.

Michelsen, J. A. 1994 Block structured multigrid solution of 2D and 3D elliptic PDE's. Tech. Rep. AFM 94-06. Dept. of Fluid Mech., Technical University of Denmark, DTU.

MikKelsen, R. F. 2003 Actuator disc methods applied to wind turbines. PhD thesis, Dept. of Fluid Mech., Technical University of Denmark, DTU.

Okulov, V. L. \& Sørensen, J. N. 2007 Stability of helical tip vortices in a rotor far wake. J. Fluid Mech. 576, 1-25.

ØYE, S. 1991 Tjæreborg wind turbine: 4. dynamic flow measurement. AFM Notat VK-204.

Prony, R. 1795 Essai expérimental et analytique. J. l'Ecole Polytech. 1 (2), 24-76.

RAO, A. R., HAMED, K. H. \& CHEN, H. L. 2003 Nonstationarities in Hydrologic and Environmental Time Series, Water Sci. and Tech. Library, vol. 45. Springer.

REMPFER, D. \& FASEL, H. F. 1994 Evolution of three-dimensional coherent structures in a flat-plate boundary layer. J. Fluid Mech. 260, 351-375.

Rowley, C. W., Mezić, I., Bagheri, S., Schlatter, P. \& Henningson, D. S. 2009 Spectral analysis of nonlinear flows. J. Fluid Mech. 641, 115-127.

Schlatter, P. \& ÖRLÜ, R. 2012 Turbulent boundary layers at moderate Reynolds numbers. Inflow length and tripping effects. J. Fluid Mech. 710, 5-34.

SCHMID, P. J. 2010 Dynamic mode decomposition of numerical and experimental data. J. Fluid Mech. 656, 5-28.

SIROVICH, L. 1987 Turbulence and the dynamics of coherent structures. I-coherent structures. IIsymmetries and transformations. III-dynamics and scaling. Q. Appl. Maths 45 (1), 561-571.

Smith, G., Schlez, W., Liddell, A., Neubert, A. \& PeÑA, A. 2006 Advanced wake model for very closely spaced turbines. In Conf. Procs. EWEC Athens. 
SøREnSEn, N. N. 1995 General purpose flow solver applied to flow over hills. PhD thesis, Ris $\varnothing$ National Laboratory, Roskilde.

Sørensen, J. N. 2011 Instability of helical tip vortices in rotor wakes. J. Fluid Mech. 682, 1-4.

Sørensen, J. N. \& VAN KUIK, G. A. M. 2011 General momentum theory for wind turbines at low tip speed ratios. Wind Energy 14 (7), 821-839.

SøRensen, J. N.\& SHEn, W. Z. 2002 Numerical modeling of wind turbine wakes. Trans. ASME: J. Fluids Engng 124 (2), 393-399.

Sørensen, J. N., Shen, W. Z. \& Munduate, X. 1998 Analysis of wake states by a full-field actuator disc model. Wind Energy 1 (2), 73-88.

TAngler, J. L., Wohlfeld, R. M. \& Miley, S. J. 1973 An experimental investigation of vortex stability, tip shapes, compressibility, and noise for hovering model rotors. NASA 2305.

TA PhuOC, L. 1994 Modèles de sous maille appliqués aux ecoulements instationnaires décollés. In Proceeding of the DRET Conference: Aérodynamique Instationnaire Turbulents - Aspects Numériques et Expérimentaux.

Troldborg, N. 2008 Actuator line modeling of wind turbine wakes. PhD thesis, Dept of Fluid Mechanics, Technical University of Denmark, DTU.

Walther, J. H., Guenot, M., Machefaux, E., Rasmussen, J. T., Chatelain, P., Okulov, V. L., Sørensen, J. N., Bergdorf, M. \& Koumoutsakos, P. 2007 A numerical study of the stabilitiy of helical vortices using vortex methods. J. Phys.: Conf. Ser. 75 (1), 012034.

WELCH, P. 1967 The use of fast Fourier transform for the estimation of power spectra: a method based on time averaging over short, modified periodograms. IEEE Trans. Audio Electroacoust. 15 (2), 70-73.

Widnall, S. E. 1972 The stability of a helical vortex filament. J. Fluid Mech. 4, 641-663. 